\title{
Estudio ANTROPOlÓGico de las ALQuerías de BeniZAhat y ZENETA (VALL D’UiXÓ, CASTELLÓN). UNA VENTANA A LA VIDA RURAL ANDALUSí
}

\author{
Anthropological study of Benizahat and Zeneta hamlets. A window into Andalusian rural life
}

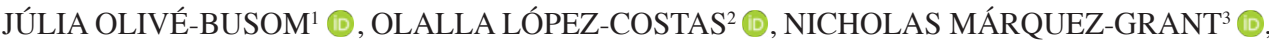 HELENA KIRCHNER ${ }^{1}$}

(1) Dpt. de Ciències de l'Antiguitat i l’Edat Mitjana. Universitat Autònoma de Barcelona.julia.olive@uab.cat , helena.kirchner@uab.cat (2) Group EcoPast - Universidade de Santiago de Compostela. Archeological Research Laboratoy - Stockholm University. Laboratorio de Antropología Física - Universidad de Granada. olalla.lopez@usc.es

(3) Cranfield Forensic Institute. Cranfield University (UK).n.marquezgrant@cranfield.ac.uk

\section{Resumen:}

En época andalusí, la Vall d'Uixó (Castellón, España) se conformaba por una serie de alquerías que estuvieron habitadas por población islámica hasta el s. XVI. A través del análisis antropológico de 99 esqueletos procedentes de dos de estas alquerías, Benizahat y Zeneta, el presente estudio explora la posible existencia de diferencias en el estilo de vida de hombres y mujeres y un posible estrés ocupacional relacionado con la economía rural en esta población. También evalúa sus condiciones de vida y niveles de estrés durante el crecimiento respecto otras poblaciones islámicas peninsulares. Aunque la población estudiada no se distingue claramente de otras poblaciones andalusíes a nivel de estrés fisiológico y ocupacional, destacan las diferencias significativas entre la población masculina y femenina en la presencia de nódulos de Schmörl y las elevadas frecuencias de caries dentales en el conjunto de la población.

Palabras clave: šarq al-Andalus, estrés fisiológico, estado de salud, arqueología islámica, cementerio.

\section{Abstract:}

During the Andalusi period, modern day Vall d'Uixó (Castellón, Spain) was formed by a series of hamlets that were inhabited by Islamic population until the 16th century. Through the anthropological analysis of 99 skeletons from two of these hamlets, Benizahat and Zeneta, this study explores the possible existence of differences in the lifestyle of males and females and occupational stress related to the rural economy of this population. In addition, their living conditions and stress levels during growth are assessed through the confrontation with other peninsular Islamic populations. Although the studied sample is not clearly distinguishable from other Andalusian populations in terms of physiological and occupational stress, the significant differences in the distribution of Schmörl's nodes between males and females and the high frequencies of dental caries in the population should be highlighted.

Key words: šarq al-Andalus, physiological stress, health status, Islamic archaeology, cemetery.

Copyright: (C) 2021 Júlia Olivé et al. This is an open access paper distributed under the terms of the 
La Vall d'Uixó (VDU) es una localidad situada en la Sierra de Espadán, al S de la provincia de Castellón (Comunitat Valenciana). Desde el s. VIII hasta el s. XIII su territorio formó parte de al-Andalus, más concretamente de šarq al-Andalus (fig. 1). Después de la conquista cristiana, que ocurrió aquí en la primera mitad del s. XIII, la población musulmana pudo permanecer en el área, como parte del Reino de Valencia, hasta el decreto de expulsión de los moriscos de 1609 , posterior a la pragmática de conversión de 1525 (Bazzana et al. 1988: 168-169; Torró 2007; 2010).

En época andalusí, esta localidad consistía en doce alquerías organizadas alrededor de un hiṣn ${ }^{1}$ situado en la cima de un cerro. A. Bazzana (1996; 1999) estudió este hiṣn y distinguió dos fases de ocupación. En la primera el hiṣn consistía en una estructura poligonal situada en el centro del asentamiento de planta pentagonal e irregular. El escaso espesor de los muros podría indicar que más que un edificio con funciones militares o defensivas se trataba de una estructura de hábitat. Este reducto iba acompañado de una muralla situada en la parte inferior del cerro, que encerraba un espacio con casas fechadas entre los ss. X y XI apoyadas en su parte interior. La calidad de sus muros indica que no se trataba de una simple

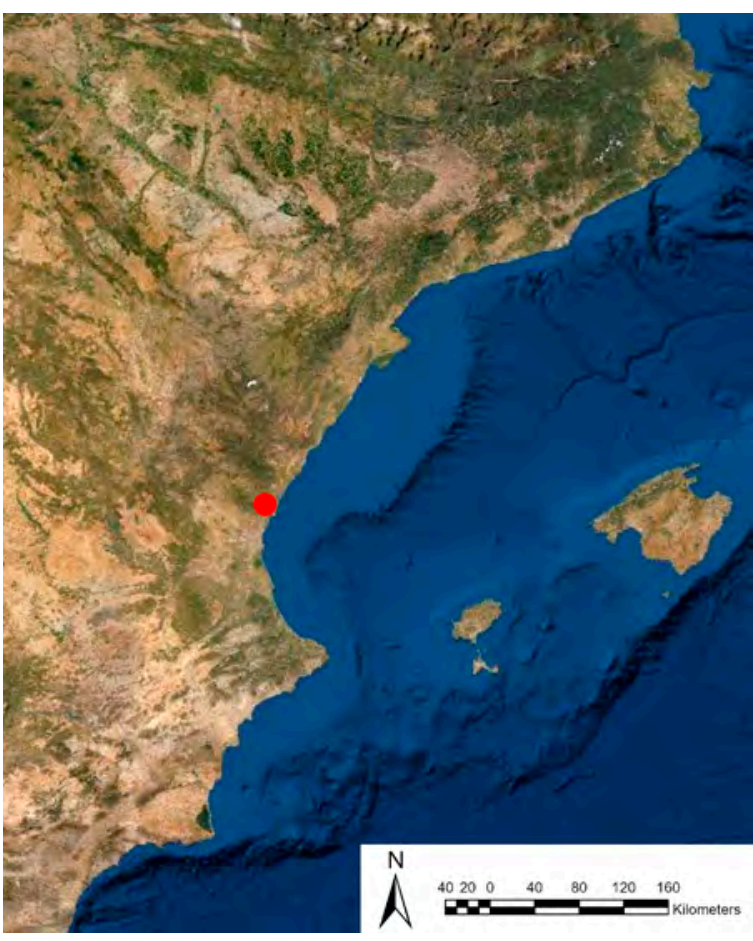

Fig. 1: Emplazamiento de la Vall d'Uixò. estructura provisional o para estabular el ganado. Bazzana propone que en la segunda fase (ss. XII-XIII) se alza el castillo actual, con el reducto inicial ya arrasado, y un recinto poligonal que encierra prácticamente todas las estructuras del castillo, con bastiones cuadrangulares y estructuras ligeras en su interior. Fuera de este complejo de fortificación se identifican zonas residenciales ocupadas durante época cristiana.

Por su parte, las doce alquerías se documentan en las fuentes feudales y se encuentran en cotas más bajas que el hiṣn. H. García García (1982) y Bazzana et al. (1988) proponen la siguiente distribución (fig. 2): Castro, Benicapdó y Alfandech se sitúan en la parte alta y angosta del valle, dentro de la Sierra de Espadán. Alcudia, Benigafull y Ceneja en diferentes puntos de una colina en la entrada del valle. Ya en el llano se encuentran Benizahat, Zeneta, Benigasló y Orleyl. Por último, se desconoce la localización de Benadalmech y Care. Mediante el estudio de la toponimia, se ha podido relacionar el poblamiento de seis de estas alquerías con el asentamiento de tribus árabes y bereberes: Benigasló, Benigafull, Zeneja, Benizahat y Zeneta podrían corresponder a los Banū Gazlūn, Gafūl, Zanāta, Sa'd y Sanhāğa respectivamente (Guichard 1976; 2016). No han sido datadas aun con exactitud o excavadas en extensión, por lo que se desconoce si todas ellas son sincrónicas.

Se tienen pocas referencias acerca del modo de vida en VDU en época andalusí. La documentación generada por la conquista feudal muestra una escasez de ganado y la existencia de cultivos como el trigo y la cebada en el s. XIII (Guichard 2001: 307). Ambos hechos coinciden con los conocimientos historiográficos que se tienen de las comunidades rurales andalusíes, las cuales parece que basaban su dieta en el consumo de cereales y legumbres (García Sánchez 2011). Sin embargo, los datos referentes a la alimentación que se pueden generar a través del estudio de la documentación feudal pueden presentar un sesgo ya que en ellos se recogen los cultivos que interesaban a los conquistadores, aquellos que se podían almacenar (Kirchner et al. 2014). En los últimos años se han iniciado estudios carpológicos y antracológicos en distintos asentamientos andalusíes (Alonso et al. 2014; Kirchner et al. 2014; Mira et al. 2017; Carrión et al. 2019; Peña-Chocarro et al. 2019) Éstos concluyen que los cereales son los más representados, principalmente trigo y cebada. También se ha documentado la presencia de legumbres y árboles frutales, especialmente en el levante. 
Tal como se ha mencionado anteriormente, VDU formaba parte de šarq al-Andalus, las tierras del oriente de al-Ándalus, que incluían el territorio de Tortosa a Almería y en el interior hasta Albarracín y Cuenca (Torró 2012). A. Bazzana y P. Guichard (1981) propusieron que la estructura del poblamiento del šarq al-Andalus se basaba en el binomio hiṣn- alquería(s) que ya se ha observado en VDU, una proposición que ha sido corroborada en esta región por varios autores (Esquilache 2018; Torró 2012; Selma 1991). Las alquerías consistían en territorios de límites no definidos con uno o diversos núcleos habitacionales ocupados por una comunidad rural. Junto a los hușūn, castillos rurales con variedad de funciones (v.g. refugio de la población o alojamiento de guarniciones), constituían la base del poblamiento rural del šarq al-Ándalus (Bazzana y Guichard 1981; Glick 2007: 4153). Tanto la arqueología como estudios toponímicos sugieren que este territorio tuvo una intensa presencia bereber junto a algunos elementos árabes desde un momento inicial (Bazzana y Guichard 1981; Bazzana et al. 1988; Guichard 2016). Diversos autores han propuesto que fueron estos grupos los que articularon las alquerías y sus espacios agrícolas (Barceló 1997; Bazzana et al. 1988; Kirchner y Navarro 1993).

Esta estructura de poblamiento cambió radicalmente con la conquista catalano-aragonesa, oficialmente completada en 1245, que dio como resultado la formación del Reino de Valencia (ver Torró 2007). A partir de 1248 se implementó una política de expulsión de comunidades andalusíes que encontró resistencia en la Sierra de Espadán, donde se identifican las aljamas de VDU y Eslida, y al S en las aljamas del valle situado entre Cocentaina y Denia (Guinot 1993; Torró 2007). Aunque parece que las aljamas que opusieron resistencia fueron aquellas protegidas por el rey -ya que los nobles propietarios de señoríos querían mantener a los musulmanes en sus tierras- tanto la expulsión de amplios segmentos poblacionales como la relocalización forzosa de otros y la mayor presión fiscal expedida sobre los musulmanes del Reino de Valencia fueron decreciendo y empobreciendo las comunidades musulmanas hasta su desaparición en el s. XVI (Guinot 1993; Guichard 2016; Torró 2007).

La permanencia de un grueso poblacional musulmán en el reino provoca una problemática considerable para la datación de las inhumaciones por rito islámico ya que se pueden encontrar desde el s. VIII hasta el s. XVI sin que sus características cambien o se puedan vincular a un periodo en particular². Además, puesto que las tumbas no

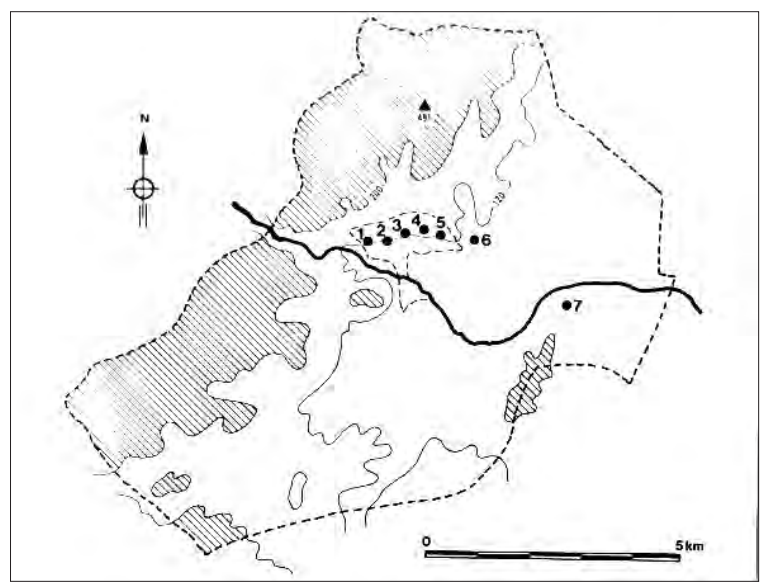

Fig. 2: Detalle del emplazamiento de las alquerías de la Vall d'Uixò planteado por Bazzana et al. (1988). El hiș̣n está marcado con un triángulo, la numeración corresponde a: 1 Benigafull; 2 Alcudia; 3 Ceneja; 4 Benizahat; 5 Zeneta; 6 Benigasló; 7 Orleyl. Fuente: Bazzana et al. 1988:160.

acostumbran a contener ajuar, las dataciones por método estratigráfico son difíciles y se requieren dataciones por ${ }^{14} \mathrm{C}$ para poder distinguir con mayor seguridad poblaciones andalusíes de aquellas que vivieron bajo dominio cristiano.

La osteoarqueología puede proveer información directa sobre las personas que formaron parte de estas comunidades en aspectos como su demografía, salud y alimentación. En la región estudiada, las poblaciones urbanas de Morella, Segorbe, Onda y Burriana han sido objeto de estudios antropológicos publicados o contenidos en memorias arqueológicas (Alfonso 2003; Barrachina 2004-05; Alfonso et al. 2006; Duarte 2009; Polo et al. 2013; Noverint 2017; Melchor y Benedito 2018). Hallazgos de cementerios andalusíes rurales, como es el caso de Forcall, Borriol y Castellón de la Plana, también han sido estudiados (Polo et al. 2008; Duarte et al. 2019; Arquer y Coch 2017). En Valencia se ha explorado en profundidad la dieta reconstruida a nivel isotópico. Alexander et al. (2015) llevaron a cabo un estudio isotópico donde se compara la alimentación de la población mudéjar de Benipeixcar y la población cristiana de Gandía, concluyendo que la población mudéjar consumía más aportes de plantas ${ }_{4} \mathrm{C}$ (mijo y sorgo) y pescado. No obstante, quedan muchos aspectos por explorar relacionados con la dieta y otros aspectos de la vida diaria de estas comunidades, en especial aquellos que caracterizan a las poblaciones rurales, cuyo estudio es todavía incipiente. 
El presente artículo plantea tres cuestiones acerca de la población rural de VDU que se estudiarán a partir de los restos óseos de 99 individuos hallados en las alquerías de Benizahat y Zeneta: 1) La posible existencia de diferencias en el estilo de vida ${ }^{3}$ de hombres y mujeres, relacionadas con las evidencias historiográficas que indican una orientación más doméstica en el caso de las mujeres. 2) La detección de un posible estrés ocupacional ${ }^{4}$ en VDU que se pueda relacionar con una economía rural, tal como sugieren otras colecciones rurales andalusíes y se infiere de varios estudios antropológicos (Novak y Šlaus 2011; Jiménez-Brobeil et al. 2012; Redfern et al. 2015). 3) La evaluación de las condiciones de vida y niveles de estrés durante el crecimiento en VDU respecto otras poblaciones islámicas peninsulares.

\section{MATERIALES Y MÉTODOS EMPLEADOS}

\section{LA NECRÓPOLIS}

Las alquerías de VDU no se han excavado en extensión, ya que se encuentran debajo de la trama del actual núcleo urbano. Por este motivo, los restos humanos estudiados proceden de varias excavaciones de urgencia realizadas entre los años 1995 y 2006 a causa de renovaciones en la canalización e instalaciones eléctricas. Estas intervenciones se concentraron en la Calle Cervantes
(1995, 2000 y 2006) y la Plaza de la Asunción (1998) y, debido a su ubicación, se atribuyen a los cementerios de las alquerías de Zeneta y Benizahat respectivamente ${ }^{5}$ (fig. 3). En conjunto, se encontraron un mínimo de 89 fosas simples, en ocasiones cubiertas por losas, que contenían individuos enterrados mediante el rito islámico. De estas 89 fosas, 81 se han atribuido al cementerio de Zeneta y 8 al de Benizahat. No se han encontrado elementos de ajuar asociados a los enterramientos. La muestra estudiada se ha datado arqueológicamente entre los ss. X y XVI (Rovira 2018). Por lo tanto, puede corresponder tanto a la población andalusí que vivió en šarq al-Andalus como a una población mudéjar que vivió después de la conquista feudal.

\section{ESTUDIO ANTROPOLÓGICO}

Se ha estimado un número mínimo de individuos de 99. Éste se ha establecido calculando el número de individuos identificados en cada fosa y sumando su total. En cada fosa, este número mínimo se calculó en base al número de los huesos repetidos que se detectaban (ej. dos húmeros izquierdos se interpretarían como la presencia de dos individuos). Se tuvieron también en cuenta diferencias de carácter sexual o de edad (White y Folkens 2005: 339-340). El índice de conservación esquelética medio de la muestra era del $42,5 \%$. Este se ha obtenido adaptando las recomendaciones de Walker et al. (1988).

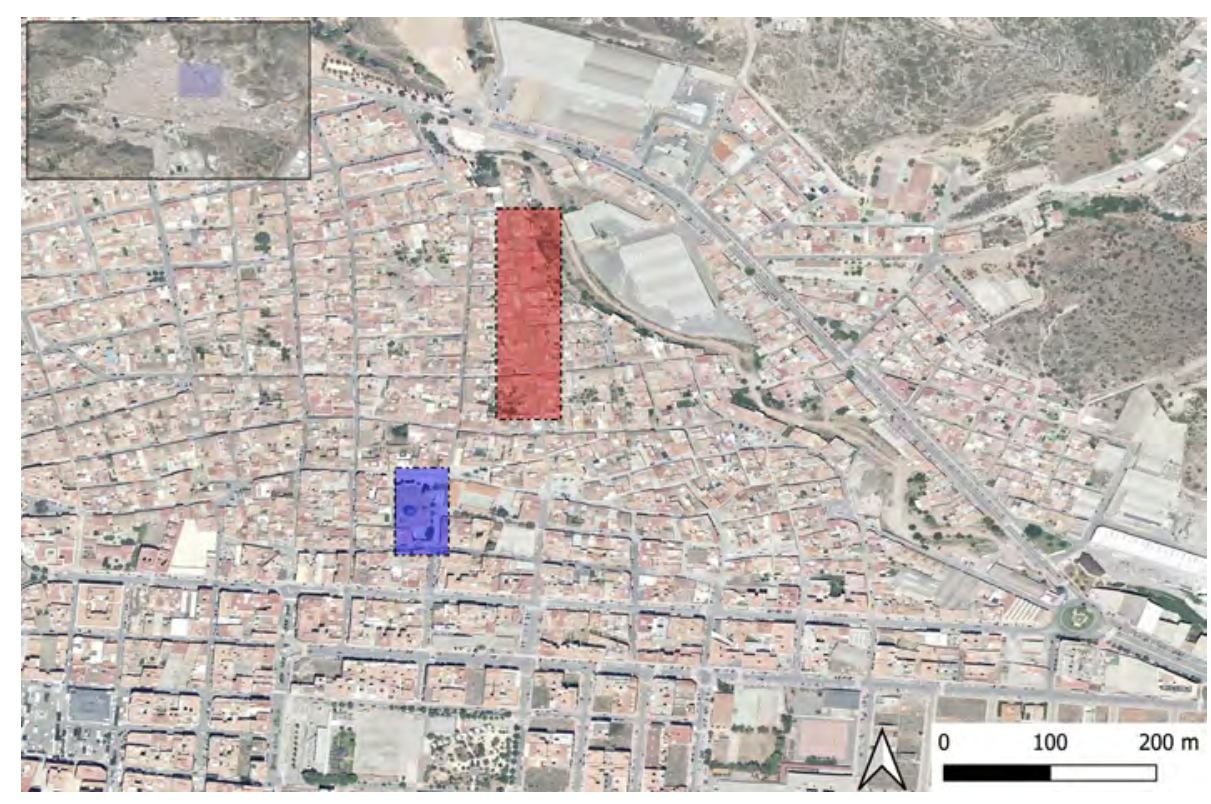

Fig. 3: Localización de las áreas de cementerio asociadas a las alquerías Zeneta (rojo) y Benizahat (azul). 
Para estimar su edad se han empleado los métodos recomendados por Schaefer y colegas (2009) en los individuos menores de 15 años, y los recomendados por Brooks y Suchey (1990), Lovejoy et al. (1985) e Işcan y Loth (1986a y b) en individuos adultos principalmente. El sexo solo se ha estimado en individuos adultos siguiendo la metodología establecida por Buikstra y Ubelaker (1994), observando los criterios para la pelvis y el cráneo.

Con el fin de conocer el estilo de vida de la comunidad de VDU a través de los restos óseos se han documentado y analizado una serie de variables antropométricas y paleopatológicas. Se incluyó la variable antropométrica de la estatura. En el caso de la paleopatología oral se registraron las caries dentales (DC). Como patologías traumáticas se documentó la presencia de nódulos de Schmörl (SN) y traumatismos. Dentro de las marcas degenerativas se documentó artrosis (OA), y en los marcadores inespecíficos de estrés metabólico la hipoplasia lineal del esmalte (LEH) y la cribra orbitalia $(\mathrm{CO})$. Estas variables fueron registradas para evaluar diferencias en el estilo de vida de hombres y mujeres (estatura, DC, SN, OA y traumatismos), en las características de la vida ocupacional rural y urbana (SN, OA y traumatismos) y la calidad de vida y posible estrés fisiológico (DC, SN, OA, traumatismos, estatura, LEH y CO).

Las diferencias intra e interpoblacionales en estatura pueden ser el resultado del entorno biocultural y se pueden atribuir a factores de estrés durante el desarrollo, aunque la mortalidad selectiva y fragilidad heterogénea pueden ser también factores determinantes (Schweich y Knüsel 2003; Vercellotti et al. 2014). Se ha estimado la estatura de cada individuo mediante las fórmulas de regresión de Pearson (1899) y Mendonça (2000) puesto que son las más indicadas para poblaciones ibéricas (Lalueza-Fox 1998). Solamente se tomaron las medidas de huesos completos y sin patologías.

Las DC tienen una etiología multifactorial y consisten en la desmineralización del esmalte dental por las bacterias bucales (Hillson 2019). En colecciones osteológicas son utilizadas para inferir diferencias en la dieta puesto que el consumo de carbohidratos puede incrementar el metabolismo bacteriano y, por lo tanto, ocasionar la aparición de DC (Grenby et al. 2001; Hillson 2019; Kubehl y Temple 2020). No obstante, no se trata de una interpretación directa y existen otros factores que pueden influir en la aparición de esta patología dental. Otros estudios sugieren que, en la población femenina, se pueden ver influidas por cambios hormonales relacionados con la fertilidad, ya que las hormonas relacionadas con el embarazo alteran el $\mathrm{pH}$ de la saliva y pueden incrementar el riesgo de desarrollar DC (Burakoff 2003; Silk et al. 2008). Se analizaron todos los dientes permanentes erupcionados para evaluar la presencia o ausencia de DC y LEH. Se excluyeron aquellos que tenían superficies bucales no observables (debido a concreciones de tierra o cálculo dental); cuando debía observarse la presencia de LEH, también se descartaron aquellos dientes que presentaban más del $50 \%$ de su área desgastada o afectada por DC. Tanto para su registro como para su valoración se emplearon estándares ampliamente utilizados en estudios antropológicos (Hillson 1996; 2001).

Los SN son el resultado de la herniación del núcleo pulposo del disco intervertebral (Waldron 2009: 45). Diversos estudios en poblaciones arqueológicas vinculan su aparición con la realización de tareas físicamente intensas y con carga mecánica, mientras que la literatura clínica los vincula a factores biomecánicos y a la realización de tareas pesadas durante la etapa de desarrollo de la vértebra (Faccia y Williams 2008; Üstündağ 2009; Dar et al. 2010; Novak y Šlaus 2011; Burke 2012). El registro sistemático de $\mathrm{SN}$ se realizó en vértebras pertenecientes a individuos adultos siguiendo los estándares descritos por Knüsel et al. (1997) descartándose aquellas vértebras cuyos discos estaban erosionados o presentaban una pérdida postmortem superior a la mitad del disco. Por otro lado, la OA consiste en la degeneración del cartílago articular. Aunque se considera que tiene un origen multifactorial y se vincula estrechamente con la edad, también se relaciona con cargas mecánicas repetitivas (Weiss y Jurmain 2007). Por lo tanto, la repetición de ciertas acciones, como aquellas realizadas en una ocupación, pueden ocasionarla (Waldron 2009: 27-28). Su registro se realizó siguiendo los criterios propuestos por Waldron (2009). Solo se consideraron esqueletos adultos y se excluyeron las epífisis erosionadas o incompletas.

El estudio de los traumatismos observados en restos humanos puede ayudar a entender las prácticas culturales y socioeconómicas, y a evaluar las condiciones ambientales y sociales de una población (Šlaus et al. 2012; Meyer y Steyn 2016). Por esta razón diversos estudios emplean frecuencias de ciertos tipos de fracturas para hacer inferencias acerca del ambiente en que una población trabaja y/o vive (e.g. Judd y Roberts 1999; Sanders et al. 2002; Djurić et al. 2006; Šlaus et al. 2012; Meyer y Steyn 2016). Las prevalencias de fracturas se calcularon tanto 
por individuo como por zona del esqueleto preservada (e.g. húmero proximal), registrando si éstas eran peri- o antemortem.

Respecto a las patologías que se desarrollan durante el crecimiento, se han estudiado LEH y CO (Scott y Hoppa 2018; Pilloud y Schwitalla 2020). LEH es un defecto del esmalte dental causado por interrupciones generalizadas en la formación de la matriz del esmalte, que se manifiestan como ranuras paralelas a la unión del cemento y el esmalte (Hillson 1996). CO consiste en porosidad en las paredes orbitales y puede variar en densidad y tamaño (Stuart-Macadam 1998). Aunque se discute la etiología de ambas, siguen considerándose indicadores válidos para estudiar la salud y el estatus nutricional durante la infancia en poblaciones pasadas (Scott y Hoppa 2018; Hillson 2019; Pilloud y Schwitalla 2020). LEH se documentó como presente cuando se observó al menos una línea o defecto en el esmalte. Para la evaluación de CO, se utilizó el sistema descrito por Stuart-Macadam (1991) anotando la presencia/ausencia de $\mathrm{CO}$ en cada órbita observada y presentándola por individuo.

Los análisis estadísticos de los datos recopilados se han hecho mediante SPSS 16. Posibles diferencias sexuales con variables categóricas se han comprobado mediante la prueba exacta de Fisher, ya que es un método adecuado para poblaciones con un número de muestra bajo. Las posibles diferencias en la estatura se analizaron mediante la Prueba U de Mann-Whitney. En ambos casos, la significancia seleccionada fue $\mathrm{p}<0,05$.

\section{RESULTADOS}

La distribución demográfica de la muestra se expresa en la Figura 4. La muestra adulta $(n=59)$ contiene más hombres $(n=26)$ que mujeres $(n=17)$. Todos los rangos de edad se encuentran representados en la muestra adulta, aunque hay un mayor número $(\mathrm{n}=18)$ de adultos indeterminados. En la población mayor de 30 años se observa un mayor número de hombres $(\mathrm{n}=13)$ que de mujeres $(\mathrm{n}=5)$. Aunque no se han encontrado individuos perinatales, destaca la proporción de individuos no adultos, cercano al $\sim 40 \%(\mathrm{n}=40)$ y con la presencia de un individuo perinatal, posiblemente propiciado por buen estado de conservación ósea de la colección (Grado 2 según McKinley 2004).

La estatura fue estimada en un total de 29 individuos obteniéndose una media de $\sim 165 \pm 4 \mathrm{~cm}$ en la población masculina y $\sim 156 \pm 7 \mathrm{~cm}$ en la población femenina (fig. $5)$. No se han observado grandes diferencias entre las estimaciones hechas por ambos métodos. Estos valores son similares a los resultados obtenidos en otros cementerios islámicos peninsulares, tanto aquellos de cronología más amplia (v.g. Maro o La Torrecilla) como los de cronologías acotadas en época andalusí (v.g. San Lázaro o Santa Teresa). Se cuenta como excepción el cementerio de La Rauda, que presenta una media para la población masculina notablemente más alta (fig. 5). El cementerio visigodo de La Almoina, también en Valencia, presenta estaturas parecidas a las obtenidas en VDU (fig. 5). Las diferencias entre sexos son significativas $(\mathrm{p}<0,05)$ tanto

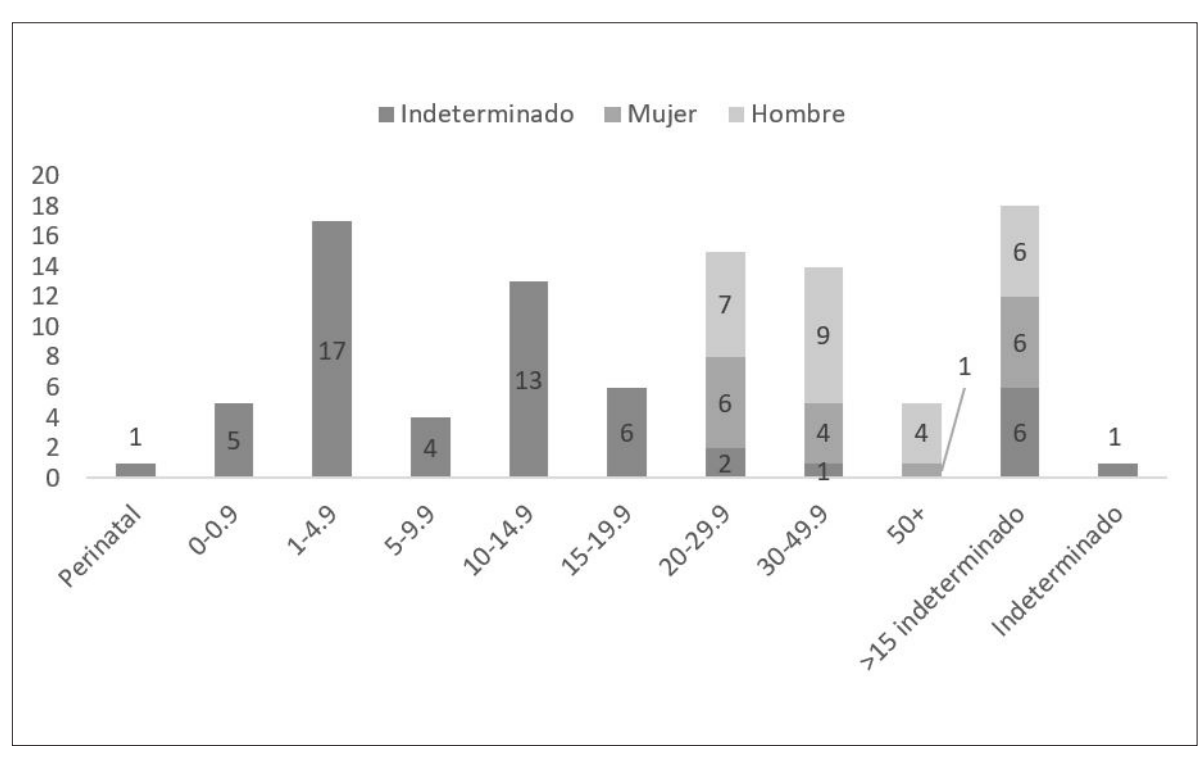

Fig. 4. Composición demográfica de la muestra. 


\begin{tabular}{|c|c|c|c|c|c|c|c|c|c|c|}
\hline & \multirow{2}{*}{ Tipo } & \multirow{2}{*}{ Ubicación } & \multicolumn{3}{|c|}{ Pearson (1899) } & \multicolumn{4}{|c|}{ Mendonça (2000) } & \multirow{2}{*}{ Autores } \\
\hline & & & Estatura media & S.D. & $p$ & Estatura media & S.D. & $p$ & $\mathrm{n}$ & \\
\hline $8 \mathrm{VDU}$ & \multirow{2}{*}{ Islámica X-XVI } & \multirow{2}{*}{ Castellón } & 165,2 & 3.9 & \multirow[b]{2}{*}{$<0,001$} & 165.5 & 4,9 & \multirow[b]{2}{*}{0,002} & 17 & \multirow{2}{*}{ Esta publicación } \\
\hline qVDU & & & 156,4 & 6.7 & & 156,5 & 7,6 & & 12 & \\
\hline ¿ Maro & \multirow{2}{*}{$\begin{array}{c}\text { Islámica VIII- } \\
\text { XV }\end{array}$} & \multirow{2}{*}{ Málaga } & $\mathrm{N} / \mathrm{A}$ & $\mathrm{N} / \mathrm{A}$ & \multirow{2}{*}{$\mathrm{N} / \mathrm{A}$} & 170,5 & 6,8 & \multirow[b]{2}{*}{$<0,001$} & $\mathrm{~N} / \mathrm{A}$ & \multirow{2}{*}{$\begin{array}{c}\text { Sánchez- Aparcero } \\
\text { et al. } 2020\end{array}$} \\
\hline q Maro & & & $\mathrm{N} / \mathrm{A}$ & $\mathrm{N} / \mathrm{A}$ & & 161,1 & 5,8 & & N/A & \\
\hline Can Fonoll & \multirow{2}{*}{ Islámica X-XIII } & \multirow{2}{*}{ Islas Baleares } & 159,4 & $\mathrm{~N} / \mathrm{A}$ & \multirow{2}{*}{$\mathrm{N} / \mathrm{A}$} & $\mathrm{N} / \mathrm{A}$ & $\mathrm{N} / \mathrm{A}$ & \multirow{2}{*}{$\mathrm{N} / \mathrm{A}$} & 4 & \multirow{2}{*}{$\begin{array}{c}\text { Kyriakou et al. } \\
2012\end{array}$} \\
\hline q Can Fonoll & & & 150.5 & $\mathrm{~N} / \mathrm{A}$ & & N/A & $\mathrm{N} / \mathrm{A}$ & & 6 & \\
\hline o San Lázaro & \multirow{2}{*}{ Islámica X-XI } & \multirow{2}{*}{ Toledo } & $\mathrm{N} / \mathrm{A}$ & $\mathrm{N} / \mathrm{A}$ & \multirow{2}{*}{$\mathrm{N} / \mathrm{A}$} & 166,7 & 7 & \multirow{2}{*}{$\mathrm{N} / \mathrm{A}$} & $\mathrm{N} / \mathrm{A}$ & \multirow{4}{*}{$\begin{array}{c}\text { Molero 2017:117- } \\
118\end{array}$} \\
\hline q San Lázaro & & & $\mathrm{N} / \mathrm{A}$ & $\mathrm{N} / \mathrm{A}$ & & 157,6 & 6 & & $\mathrm{~N} / \mathrm{A}$ & \\
\hline S Santa Teresa & \multirow{2}{*}{ Islámica VIII-X } & \multirow{2}{*}{ Toledo } & $\mathrm{N} / \mathrm{A}$ & $\mathrm{N} / \mathrm{A}$ & \multirow{2}{*}{$\mathrm{N} / \mathrm{A}$} & 163,8 & 7 & \multirow{2}{*}{ N/A } & N/A & \\
\hline \% Santa Teresa & & & $\mathrm{N} / \mathrm{A}$ & $\mathrm{N} / \mathrm{A}$ & & 156,3 & 6 & & $\mathrm{~N} / \mathrm{A}$ & \\
\hline c La Almoina & \multirow{2}{*}{ Visigoda VI-X } & \multirow{2}{*}{ Valencia } & 1,69 & $\mathrm{~N} / \mathrm{A}$ & \multirow{2}{*}{$\mathrm{N} / \mathrm{A}$} & $\mathrm{N} / \mathrm{A}$ & $\mathrm{N} / \mathrm{A}$ & \multirow{2}{*}{$\mathrm{N} / \mathrm{A}$} & 7 & \multirow{4}{*}{ Djilali 2018} \\
\hline q La Almoina & & & 1,57 & $\mathrm{~N} / \mathrm{A}$ & & N/A & $\mathrm{N} / \mathrm{A}$ & & 7 & \\
\hline ¿ La Rauda & \multirow{2}{*}{$\begin{array}{l}\text { Islámica XI- } \\
\text { XIII }\end{array}$} & Yono. & 1,74 & $\mathrm{~N} / \mathrm{A}$ & Nit & $\mathrm{N} / \mathrm{A}$ & $\mathrm{N} / \mathrm{A}$ & No & 9 & \\
\hline \& La Rauda & & varticta & 1,56 & $\mathrm{~N} / \mathrm{A}$ & 10,21 & N/A & $\mathrm{N} / \mathrm{A}$ & $10 / 1$ & 9 & \\
\hline o La Torrecilla & Islámica IX- & Gronod & 164,9 & 9 & $\mathrm{~N} / \mathrm{A}$ & $\mathrm{N} / \mathrm{A}$ & $\mathrm{N} / \mathrm{A}$ & $N / A$ & $\mathrm{~N} / \mathrm{A}$ & Souich 1974 \\
\hline q La Torrecilla & XIV & & 151,8 & 9 & & N/A & N/A & & N/A & \\
\hline
\end{tabular}

Fig. 5: Estaturas medias en la muestra estudiada y comparación con otras colecciones.

si se emplean las fórmulas de regresión de Mendonça (2000) como las de Pearson (1899).

Las caries dentales (DC) fueron evaluadas en un total de 592 dientes pertenecientes a 46 individuos. La frecuencia total estimada en la población adulta es del $21,1 \%$. Como suele ser habitual, la dentición posterior se ve más afectada. No se encuentran diferencias significativas entre hombres $(23,1 \%)$ y mujeres $(22,8 \%)$ (fig. 6$)$. Se debe advertir, sin embargo, que el número de dientes disponibles en la población femenina es inferior, 171 contra 281 en la masculina.

El mayor número de individuos masculinos mayores de 30 años $(n=13)$ respecto a los de sexo femenino $(n=5)$ podría estar condicionando los valores observados puesto que vivir más puede aumentar el riesgo de desarrollar DC. Por este motivo, en la Figura 7 se muestran las frecuencias de DC en dos grupos de edad (grupo1: $<30$ años; grupo2: $>30$ años). Se puede observar que el grupo de mayor edad no presenta una tendencia clara de frecuencias más altas, sin obtenerse resultados significativos en ninguno de los casos.

Los nódulos de Schmörl (SN) son notablemente menos frecuentes en individuos femeninos $(6,8 \%)$ que en masculinos $(28,5 \%)$ y las diferencias encontradas resultan significativas ( $\mathrm{p}<0,001$; fig. 8). Se debe advertir que el número de observaciones realizadas es siempre superior en los individuos masculinos. La mayoría de articulaciones muestran signos de artrosis (OA), con una afectación total del $\sim 6 \%$. Parece ser más común en las extremidades superiores $(7,7 \% ; 1,7 \%$ en las inferiores) y no se aprecian diferencias significativas entre sexos. La OA vertebral presenta diferencias significativas entre sexos con $\mathrm{p}<0,001$ puesto que los hombres $(16,2 \%)$ presentan frecuencias más elevadas que las mujeres $(2,6 \%)$ (fig. 8). Otra vez, debe tenerse en cuenta que el número de observaciones realizadas en la población femenina es inferior.

Puesto que la mayoría de los individuos mayores de 30 años son hombres, es posible que esto condicione las observaciones de SN y OA. Debido a ello, se ha establecido una comparación entre aquellos individuos menores de 30 años y los de edad superior (fig. 9). Los individuos del grupo joven presentan frecuencias mayores de $\mathrm{SN}$ en ambos grupos vertebrales observados, pero las diferencias solamente resultan significativas cuando se tiene en cuenta el total de observaciones $(\mathrm{p}=0,03)$. Ninguno de los individuos menores de 30 años muestra signos de $\mathrm{OA}$ en los huesos largos. Salvo en la articulación de la cadera y 


\begin{tabular}{|l|c|c|c|c|c|c|c|c|c|c|}
\hline & \multicolumn{3}{|c|}{ Total DC $(\mathrm{n}=46)$} & \multicolumn{3}{c|}{ Hombres (n=23) } & \multicolumn{3}{c|}{ Mujeres (n=14) } & \% \\
\hline & $\mathrm{n}$ & $\mathrm{N}$ & $\%$ & $\mathrm{n}$ & $\mathrm{N}$ & $\%$ & $\mathrm{n}$ & $\mathrm{N}$ & $\%$ \\
\hline I1 & 8 & 85 & 9 & 2 & 40 & 5 & 4 & 24 & 17 & 0,17 \\
\hline I2 & 7 & 86 & 8 & 4 & 47 & 9 & $\mathrm{I}$ & 26 & 4 & 0,65 \\
\hline C & 9 & 99 & 9 & 3 & 49 & 6 & 5 & 29 & 17 & 0,14 \\
\hline P1 & 27 & 91 & 30 & 13 & 32 & 41 & 10 & 30 & 33 & 0,61 \\
\hline P2 & 17 & 85 & 20 & 10 & 38 & 26 & 5 & 26 & 19 & 0,56 \\
\hline M1 & 23 & 52 & 44 & 13 & 24 & 54 & 6 & 12 & 50 & 1 \\
\hline M2 & 20 & 48 & 42 & 13 & 26 & 50 & 3 & 9 & 33 & 0,46 \\
\hline M3 & 14 & 46 & 30 & 7 & 25 & 28 & 5 & 15 & 33 & 0,74 \\
\hline TOTAL & 125 & 592 & 21,1 & 65 & 281 & 23,1 & 39 & 171 & 22,8 & 1 \\
\hline
\end{tabular}

Fig. 6: Frecuencias de caries dentales (DC) en la muestra estudiada por diente observado. Al no encontrarse diferencias significativas entre lateralidades, se han agrupado.

\begin{tabular}{|l|c|c|c|c|c|c|c|}
\hline & \multicolumn{3}{|c|}{$<30$ años } & \multicolumn{3}{c|}{$>30$ años } & N \\
\hline & $\mathrm{n}$ & $\mathrm{N}$ & $\%$ & $\mathrm{n}$ & $\mathrm{N}$ & $\mathrm{p}$ \\
\hline $\mathrm{I1}$ & 6 & 38 & 16 & 0 & 25 & 0 & 0,07 \\
\hline $\mathrm{I} 2$ & 3 & 44 & 7 & 3 & 29 & 10 & 0,68 \\
\hline C & 7 & 47 & 15 & 2 & 28 & 7 & 0,47 \\
\hline P1 & 14 & 42 & 33 & 10 & 27 & 37 & 0,8 \\
\hline P2 & 9 & 41 & 22 & 6 & 25 & 24 & 1 \\
\hline M1 & 11 & 25 & 44 & 7 & 15 & 47 & 1 \\
\hline M2 & 11 & 24 & 46 & 7 & 13 & 54 & 0,74 \\
\hline M3 & 10 & 27 & 37 & 3 & 11 & 27 & 0,71 \\
\hline TOTAL & 71 & 288 & 25 & 38 & 173 & 22 & 0,57 \\
\hline
\end{tabular}

Fig. 7: Frecuencias de caries dentales (DC) en la muestra estudiada por diente observado. Al no encontrarse diferencias significativas entre lateralidades, se han agrupado.

\begin{tabular}{|c|c|c|c|c|c|c|c|c|c|c|}
\hline & \multicolumn{3}{|c|}{ Total } & \multicolumn{3}{|c|}{ Mujeres } & \multicolumn{3}{|c|}{ Hombres } & \multirow[b]{2}{*}{$p$} \\
\hline & $\mathrm{n}$ & $\mathrm{N}$ & $\%$ & $\mathrm{n}$ & $\mathrm{N}$ & $\%$ & $\mathrm{n}$ & $\mathrm{N}$ & $\%$ & \\
\hline OA extremidad superior & 36 & 468 & 7,7 & 7 & 138 & 5,1 & 25 & 282 & 8,9 & 0,24 \\
\hline Hombro & 2 & 172 & 1,2 & 3 & 48 & 6 & 16 & 110 & 14,5 & 0,19 \\
\hline Codo & 9 & 193 & 4.7 & 4 & 64 & 6 & 4 & 109 & 3,7 & 0,47 \\
\hline Muñeca & 7 & 103 & 6,8 & 0 & 28 & 0 & 5 & 63 & 8 & 0,32 \\
\hline OA extremidad inferior & 8 & 465 & 1,7 & 4 & 143 & 2,8 & 3 & 256 & 1,2 & 0,26 \\
\hline Cadera & 2 & 145 & 1,4 & 2 & 46 & 4 & 0 & 83 & 0 & 0,13 \\
\hline Rodilla & 5 & 202 & 2,5 & 1 & 64 & 4 & 3 & 106 & 2,8 & 1 \\
\hline Tobillo & 1 & 118 & 0,8 & 1 & 33 & 4 & 0 & 67 & 0 & 0,33 \\
\hline OA Vértebras & 145 & 1103 & 13,1 & 9 & 344 & 2,6 & 110 & 677 & 16,2 & $<0,001$ \\
\hline SN vértebras torácicas & 92 & 753 & 12,2 & 10 & 156 & 6,4 & 57 & 230 & 24,8 & $<0,001$ \\
\hline SN vértebras lumbares & 58 & 275 & 21,1 & 2 & 20 & 10 & 35 & 93 & 37,6 & 0,018 \\
\hline SN total & 150 & 1028 & 14,6 & 12 & 176 & 6,8 & 92 & 323 & 28,5 & $<0,001$ \\
\hline
\end{tabular}

Fig. 8: Prevalencias y frecuencias de artrosis (OA) y nódulos de Schmörl (SN) en la muestra estudiada. En OA las lateralidades se encuentran agrupadas al no encontrarse diferencias significativas ${ }^{6}$. 


\begin{tabular}{|l|c|c|c|c|c|c|c|}
\hline & \multicolumn{3}{|c|}{$<30$ años } & \multicolumn{3}{|c|}{$>30$ años } & N \\
\hline & $\mathrm{n}$ & $\mathrm{N}$ & $\%$ & $\mathrm{n}$ & $\mathrm{N}$ & $\mathrm{p}$ \\
\hline OA extremidad superior & 0 & 149 & 0 & 19 & 203 & 9,4 & 0 \\
\hline Hombro & 0 & 36 & 0 & 12 & 84 & 14 & 0 \\
\hline Codo & 0 & 72 & 0 & 2 & 77 & 3 & 0 \\
\hline Muñeca & 0 & 41 & 0 & 5 & 42 & 12 & 0 \\
\hline OA extremidad inferior & 0 & 169 & 0 & 5 & 175 & 2,9 & 0 \\
\hline Cadera & 0 & 51 & 0 & 0 & 58 & 0 & 1 \\
\hline Rodilla & 0 & 73 & 0 & 5 & 75 & 7 & 0 \\
\hline Tobillo & 0 & 45 & 0 & 0 & 42 & 0 & 1 \\
\hline OA vertebral & 13 & 447 & 2,9 & 116 & 509 & 22,8 & $<0,001$ \\
\hline SN vértebras torácicas & 48 & 228 & 21,1 & 39 & 264 & 14,8 & 0,08 \\
\hline SN vértebras lumbares & 27 & 101 & 26,7 & 20 & 104 & 19,2 & 0,25 \\
\hline SN & 75 & 329 & 22,8 & 59 & 368 & 16 & 0,03 \\
\hline
\end{tabular}

Fig. 9: Prevalencias y frecuencias de artrosis (OA) y nódulos de Schmörl (SN) en la muestra estudiada por grupos de edad. En OA las lateralidades se encuentran agrupadas.

\begin{tabular}{|l|c|c|c|c|c|c|c|c|c|c|c|c|}
\hline & \multicolumn{3}{|c|}{ Hombres <30 } & \multicolumn{3}{c|}{ Mujeres <30 } & \multicolumn{3}{c|}{ Hombres $>30$} & \multicolumn{3}{c|}{ Mujeres $>30$} \\
\hline & $\mathrm{n}$ & $\mathrm{N}$ & $\%$ & $\mathrm{n}$ & $\mathrm{N}$ & $\%$ & $\mathrm{n}$ & $\mathrm{N}$ & $\%$ & $\mathrm{n}$ & $\mathrm{N}$ & $\%$ \\
\hline OA extremidad superior & 0 & 89 & 0 & 0 & 63 & 0 & 16 & 139 & 11,5 & 1 & 46 & 2 \\
\hline OA extremidad inferior & 0 & 87 & 0 & 0 & 54 & 0 & 3 & 129 & 2,3 & 1 & 33 & 0 \\
\hline VOA & 4 & 228 & 1,8 & 9 & 187 & 4,8 & 96 & 330 & 29,1 & 36 & 253 & 14,2 \\
\hline SN & 53 & 188 & 28 & 8 & 108 & 7 & 39 & 273 & 14 & 2 & 67 & 3 \\
\hline
\end{tabular}

Fig. 10: Prevalencias y frecuencias de artrosis (OA) y nódulos de Schmörl (SN) en la muestra adulta estudiada por grupos de sexo y edad. En OA las lateralidades se encuentran agrupadas.

el tobillo, las frecuencias más elevadas en el grupo de mayores de 30 años se presentan como significativas (fig. 9). En el caso de la OA vertebral, aunque el grupo joven presenta esta patología $(2,9 \%)$, la frecuencia observada es mucho menor que el grupo de mayores de 30 años $(22,8 \%)$ y la diferencia resulta significativa $(>0,001)$.

Si la muestra estudiada se divide por sexos y los grupos de edad especificados anteriormente, los hombres muestran frecuencias mayores independientemente del grupo de edad (fig. 10). El grupo joven, independientemente del sexo, no muestra OA en las extremidades superiores o inferiores, y una frecuencia del 2,9\% en las vértebras de individuos masculinos y del $4,8 \%$ en femeninos. La OA vertebral en individuos jóvenes es el único caso en que esta patología es más usual en mujeres que en hombres. Con todo, es posible que la edad, con una mayoría de mujeres jóvenes y hombres mayores de 30 años, sea un factor que influye y condiciona la supuesta diferencia significativa entre sexos observada en la Figura 8.
No se han encontrado fracturas perimortem en la muestra, pero sí nueve casos de fracturas antemortem en ocho individuos distintos ( $\mathrm{n}=8 / 99 ; \sim 8 \%$ ) (fig. 11). Dos individuos presentan fracturas costales, otros dos mandibulares en su plano anterior, y se cuentan dos fracturas claviculares. Otros dos individuos presentan una fractura de peroné y metacarpo respectivamente.

No se observa hipoplasia del esmalte (LEH) en la dentición decidua, mientras que en la dentición permanente se presenta con una frecuencia total del $37,1 \%$ (fig. 12). Parece ser ligeramente más abundante en hombres, sin que las diferencias sean significativas $(p=0,22)$. En los 14 individuos no adultos analizados, más de la mitad tienen marcas de cribra orbitalia (CO; 57\%), mientras que la muestra adulta presenta una frecuencia del 17\% (fig. 12). Ninguno de los individuos femeninos $(\mathrm{n}=10)$ tiene marcas de $\mathrm{CO}$, a diferencia de los masculinos, que presentan una frecuencia del 18\% (17 individuos). Por lo general, se observó una porosidad de grado medio-leve correspondiente a los grados 1-2 propuestos por Stuart-Macadam (1991). 


\begin{tabular}{|c|c|c|c|c|c|}
\hline Individuo & Sexo & Edad (años) & Localización & $\mathrm{n} / \mathrm{N}$ & $\%$ \\
\hline $\mathrm{AClF} 3$ & Indet. & $20-29,9$ & Tercer metatarso derecho. & $1 / 226$ & $<1$ \\
\hline AC7F5 & Mujer & $20-29,9$ & Costilla izquierda. & $1 / 275$ & $<1$ \\
\hline C95F30 & Hombre & $50+$ & Costilla derecha. & $1 / 272$ & $<1$ \\
\hline C95F21 & Hombre & $20-29,9$ & Tercio distal del peroné derecho. & $1 / 52$ & 2 \\
\hline CO0UE117 & Mujer & $>15$ & Mandíbula anterior. & $2 / 53$ & 4 \\
\hline C95F36 & Mujer & $20-29,9$ & Clavícula acromial izquierda. & $1 / 35$ & 3 \\
\hline $\mathrm{C} 06 \mathrm{~F} 3$ & Hombre & $30-49,9$ & $\begin{array}{l}\text { Mandíbula anterior. Clavícula } \\
\text { acromial derecha. }\end{array}$ & $2 / 53 ; 1 / 37$ & $4 ; 3$ \\
\hline $\mathrm{C} 06 \mathrm{~F} 21$ & Indet. & $30-49,9$ & Quinto metacarpo derecho. & $1 / 219$ & $<1$ \\
\hline
\end{tabular}

Fig. 11: Descripción y frecuencia de las fracturas antemortem encontradas en la muestra.

\begin{tabular}{|c|c|c|c|c|c|c|c|c|c|c|c|c|c|}
\hline & \multicolumn{3}{|c|}{ Mayores de 15} & \multicolumn{3}{|c|}{ Hombres } & \multicolumn{3}{|c|}{ Mujeres } & & \multicolumn{3}{|c|}{ Menores de $15^{*}$} \\
\hline & $\mathrm{n}$ & $\mathrm{N}$ & $\%$ & $\mathrm{n}$ & $\mathrm{N}$ & $\%$ & $\mathrm{n}$ & $\mathrm{N}$ & $\%$ & $p$ & $\mathrm{n}$ & $\mathrm{N}$ & $\%$ \\
\hline LEH anterior (6) & 222 & 372 & 59,7 & 72 & 129 & 55,8 & 39 & 81 & 48,1 & 0,32 & 0 & 97 & 0 \\
\hline LEH posterior & 82 & 447 & 18,3 & 32 & 148 & 21,6 & 12 & 81 & 14,8 & 0,23 & 0 & 69 & 0 \\
\hline LEH total & 304 & 819 & 37,1 & 104 & 277 & 37,6 & 51 & 162 & 31,5 & 0,22 & 0 & 166 & 0 \\
\hline $\mathrm{CO}$ & 6 & 34 & 17 & 3 & 17 & 18 & 0 & 10 & 0 & 0,27 & 8 & 14 & 57 \\
\hline
\end{tabular}

Fig. 12: Frecuencias de hipoplasia de esmalte (LEH) y criba orbitalia (CO) en la muestra estudiada. *Se muestra la dentición decidua.

\section{DISCUSIÓN}

A nivel demográfico, no se encuentra una distribución anormal que indique un episodio de mortalidad excepcional o catastrófica (DeWitte 2010; Curtis y Roosen 2017). La muestra no adulta presenta mayoritariamente individuos de entre 1 y 5 años $(\sim 44 \%)$, seguida de individuos de 10 a 15 años (13/39). Los individuos de 5 a 10 años $(\sim 10 \%)$ y de 0 a 1 años $(\sim 13 \%)$ tienen una representación menor, encontrándose un solo individuo perinatal (fig. 2). Por lo tanto, la distribución de la mortalidad no-adulta coincide en gran medida con los patrones de mortalidad de poblaciones preindustriales ya que desciende después de los 5 años y vuelve a aumentar en el rango de 10 a 15 años (Rousham y Humphrey 2002: 124). Sin embargo, también cabría esperar una mortalidad mayor en los individuos de menos de 1 año (Rousham y Humphrey 2002: 124). La baja representación de este grupo se puede deber al número de muestra, a la fragilidad de los restos, y al hecho que solo una parte del cementerio ha sido excavado, por lo que se cuenta con una muestra parcial de la población (Chamberlain 2009).

\section{DIFERENCIAS EN EL ESTILO DE VIDA DE HOM- BRES Y MUJERES}

Se ha detectado un dimorfismo sexual significativo en la estimación de la talla media por sexo, puesto que la estatura estimada para los hombres es $\sim 9 \mathrm{~cm}$ más de media que la de las mujeres (fig. 5). Este valor es considerado normal por Manouvrier (1893) y se ajusta a los rangos observados en poblaciones modernas (Bogin et al. 2017). Se observan estaturas muy similares en otras poblaciones islámicas peninsulares, salvo en la necrópolis de la Rauda, donde la población masculina es notablemente más alta ( $\sim 9 \mathrm{~cm}$; fig. 13) (Djilali 2018). La frecuencia de caries dentales (DC) difiere entre hombres y mujeres sin que estas diferencias sean significativas o constantes (fig. 6). Tal y como muestra la Figura 7, 
Fig. 13: Localización de los cementerios islámicos mencionados en la discusión: 1. Verge de Lledó (Castelló); 2. Burriana y Vall d'Uixó; 3. la Rauda y la Almoina (Valencia); 4. Xàtiva; 5. Can Fonoll; 6. Marroquíes Bajos; 7. Maro; 8. San Nicolás; 9. el Fontanar; 10. la Torreci1la; 11. San Lázaro y Santa Teresa (Toledo); 12. Santarem.

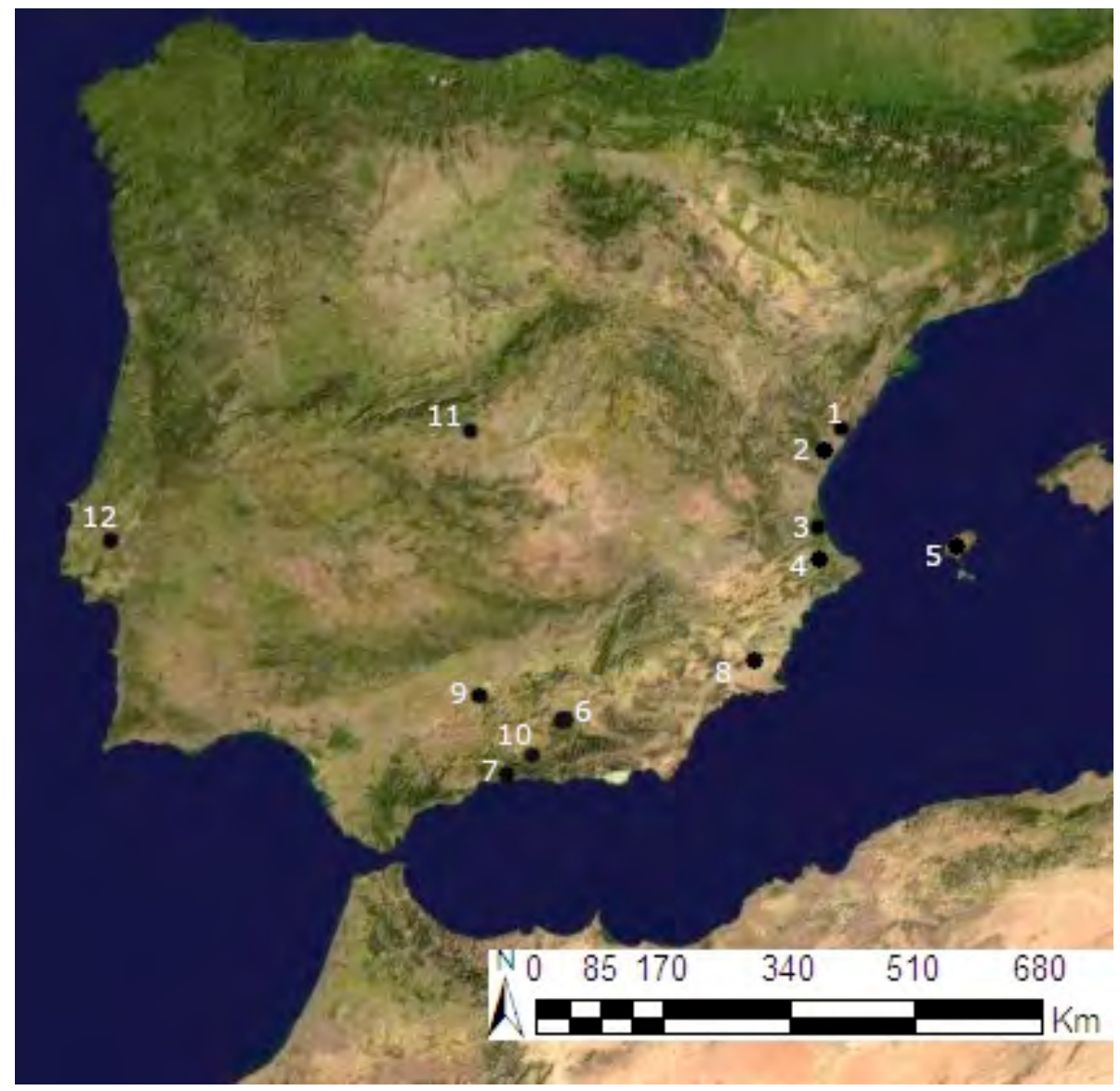

la edad no parece ser un factor que haya influenciado los resultados obtenidos por sexos. Por lo tanto, estos parámetros no permiten plantear posibles diferencias de alimentación. En otras necrópolis islámicas peninsulares, donde estas diferencias también se han analizado, se han obtenido resultados similares (Brandi 1992: 124126; López-Morango 2020: 97-117).

La población femenina presenta frecuencias significativamente más bajas de nódulos de Schmörl (SN) (fig. $8)$. Este patrón se repite en otras necrópolis andalusíes salvo en el Fontanar, donde la población femenina muestra una mayor afectación (fig. 14) (Lacalle y Guijo 2006; Aran 2012). La colección analizada está compuesta por un mayor número de hombres mayores de 30 años que de mujeres. Esto podría influenciar las diferencias detectadas por sexo. Sin embargo, si la muestra estudiada se divide en grupos de edad (grupo 1: 15-30 años; grupo 2: >30 años), las diferencias observadas no resultan significativas. Solo cuando se agrupan ambos grupos vertebrales lo son y se destaca que los SN son más comunes en los individuos más jóvenes (fig. 9). Además, los datos de la Figura 10 parecen indicar que, independientemente del grupo de edad, los individuos masculinos tienden a presentar frecuencias más elevadas de SN.

La artrosis (OA) vertebral es significativamente más común en hombres, lo que también se detecta en la necrópolis de la Torrecilla, pero no en la de Maro (fig. 14), ambas rurales (Jiménez-Brobeil et al. 2012; Sánchez-Aparcero et al. 2020). No se observan diferencias significativas en las otras articulaciones, aunque parece intuirse que la población masculina presenta frecuencias más elevadas en las articulaciones de miembros superiores y la femenina en las de miembros inferiores. No obstante, la mayor cantidad de individuos masculinos de edad superior a 30 años podría estar condicionando los resultados obtenidos en esta patología degenerativa estrechamente ligada a la edad. De hecho, se obtienen diferencias significativas, siendo OA más frecuente entre los mayores de 30 años 


\begin{tabular}{|c|c|c|c|c|c|c|c|c|}
\hline Yacimiento & Ubicación & Cronología & Tipo & Total* & No-adultos ${ }^{* *}$ & Hombres & Mujeres & Autores \\
\hline \multicolumn{9}{|c|}{$D C$} \\
\hline VDU & Castellón & ss. X-XVl & Rural & $21,10 \%$ & $5 \%$ & $23,10 \%$ & $22,80 \%$ & N/A \\
\hline Xàtiva & Valencia & s. $X$ ? & Rural & $16 \%$ & - & - & - & Negre et al. 2003 \\
\hline El Fontanar & Córdoba & s. $X$ & Urbano & $9 \%$ & - & - & - & $\begin{array}{l}\text { Lacalle y Guijo } \\
2006\end{array}$ \\
\hline San Nicolás & Murcia & ss. XI-XIII & Urbano & - & $5,30 \%$ & $14,6 \%$ & $18,7 \%$ & Brandi 1992 \\
\hline \multicolumn{9}{|c|}{$S N$} \\
\hline VDU & Castellón & ss. X-XVI & Rural & $14,60 \%$ & $\mathrm{~N} / \mathrm{A}$ & $28,50 \%$ & $6,80 \%$ & $\mathrm{~N} / \mathrm{A}$ \\
\hline La Torrecilla & Granada & ss. IX-XIV & Rural & - & - & $35,9 \%$ & $8,6 \%$ & $\begin{array}{l}\text { Jiménez-Brobeil et } \\
\text { al. } 2012\end{array}$ \\
\hline Santarém & Portugal & ss. VIII-XII & Urbano & $9 \%$ & - & - & - & Rodrigues 2013 \\
\hline San Nicolás & Murcia & ss. XI-XIII & Urbano & $12,10 \%$ & - & $23,3 \%$ & $19 \%$ & $\begin{array}{l}\text { Campo 2015:149- } \\
151\end{array}$ \\
\hline \multicolumn{9}{|c|}{$O A$} \\
\hline VDU & Castellón & ss. X-XVI & Rural & $6 \%$ & $\mathrm{~N} / \mathrm{A}$ & $\mathrm{N} / \mathrm{A}$ & $\mathrm{N} / \mathrm{A}$ & N/A \\
\hline La Torrecilla & Granada & ss. IX-XIV & Rural & - & - & $13,1 \%$ **** & $6,7 \%$ * * & $\begin{array}{l}\text { Jiménez-Brobeil et } \\
\text { al. } 2012\end{array}$ \\
\hline \multicolumn{9}{|c|}{ Fracturas } \\
\hline VDU & & ss. X-XVI & Rural & $8 \%$ & $\mathrm{~N} / \mathrm{A}$ & $\mathrm{N} / \mathrm{A}$ & $\mathrm{N} / \mathrm{A}$ & N/A \\
\hline Marroquíes Bajos & Jaén & ss. IX-XIII & Rural & $9 \%$ & - & - & - & Aran 2012 \\
\hline El Fontanar & Córdoba & s. $X$ & Urbano & $\sim 15 \%$ & - & $\sim 10 \%$ & $\sim 20 \%$ & $\begin{array}{l}\text { Lacalle y Guijo } \\
2006\end{array}$ \\
\hline \multicolumn{9}{|c|}{$\mathrm{CO}$} \\
\hline VDU & Castellón & ss. X-XVI & Rural & $17 \%$ & $57 \%$ & $18 \%$ & $0 \%$ & N/A \\
\hline Burriana & Castellón & ss. XI-XIII & Urbano & $42,80 \%$ & - & - & - & Polo et al. 2013 \\
\hline Verge de Lledó & Valencia & ss. XI-XIII & Rural? & $37,9 \%$ & & & & Polo et al. 2008 \\
\hline Maro & Málaga & ss. VIII-XV & Rural & $9,1 \%$ & - & - & - & $\begin{array}{l}\text { Sánchez-Aparcero } \\
\text { et al. } 2020\end{array}$ \\
\hline El Fontanar & Córdoba & s. X & Urbano & - & $4 \%$ & $2,60 \%$ & $2 \%$ & $\begin{array}{l}\text { Lacalle y Guijo } \\
2006\end{array}$ \\
\hline \multicolumn{9}{|c|}{$L E H$} \\
\hline VDU & Castellón & ss. X-XVI & Rural & 37,1 & 0 & $37,60 \%$ & $31,50 \%$ & $\mathrm{~N} / \mathrm{A}$ \\
\hline Xàtiva & Valencia & s. $X$ ? & Rural & $14 \%$ & - & - & - & Negre et al. 2003 \\
\hline El Fontanar & Córdoba & s. $X$ & Urbano & $25 \%$ & - & - & - & $\begin{array}{l}\text { Lacalle y Guijo } \\
2006\end{array}$ \\
\hline San Nicolás & Murcia & ss. XI-XIII & Urbano & - & $2,7 \%$ & $29 \%$ & $29,7 \%$ & Brandi 1992 \\
\hline
\end{tabular}

Fig. 14: Tabla resumen de las frecuencias en patologías obtenidas en otros sitios islámicos. *refiere a los adultos ** en dentición refiere a la decidua $* * *$ solamente artrosis $(\mathrm{OA})$ vertebral. 
(fig. 9). Los resultados obtenidos al dividir la muestra teniendo en cuenta el sexo y la edad de los individuos parecen confirmar esta tendencia (fig. 10). Por lo tanto, es posible que la mayor cantidad de individuos de sexo masculino influya en los resultados obtenidos analizando la población en conjunto.

Por otro lado, no parece que el sexo juegue un papel en la distribución de las fracturas ante mortem. Las fracturas costales, claviculares, de metacarpos y de metatarsos suelen ser el resultado de caídas y accidentes (fig. 11) (Lovell y Grauer 2019). Aunque las fracturas mandibulares y otras fracturas del esqueleto facial pueden ser el resultado de violencia interpersonal, también pueden ser causadas por caídas y otro tipo de accidentes que causen un impacto directo (Martin y Harrod 2015; Lovell y Grauer 2019).

Asumiendo el sexo como una aproximación al género de los individuos, parece que la mayoría de los marcadores estudiados no presentan una diferencia entre hombres y mujeres. Los resultados obtenidos en SN podrían sugerir una diferenciación sexual de la actividad física. En el caso de los hombres las frecuencias observadas son equiparables a las publicadas para poblaciones modernas de las que se conoce la realización de tareas físicamente pesadas y exigentes (Burke 2012, reporta un $\sim 19 \%$ en SN una población militar norteamericana). No se puede concluir, por lo tanto, que con los datos obtenidos la división de la actividad coincida con los datos historiográficos. Según éstos, la mujer se dedicaba principalmente a las tareas domésticas y el cuidado de los niños, mientras que la población masculina se encargaba preferiblemente del resto de sectores económicos (Ávila 1995; Cabanillas 2012; Pérez Ordóñez 2018). Aun así, hay muy poca información acerca de la mujer en el mundo rural y se ha propuesto que, paralelamente a las tareas domésticas, podría desarrollar tareas similares a los hombres (Shatzmiller 1993: 348350). A nivel antropológico no hay ningún estudio que integre y compare los datos obtenidos en diferentes poblaciones andalusíes, cuyos números muestrales son bajos una vez se dividen en grupos de acuerdo con el sexo y edad de los individuos.

\section{ESTRÉS OCUPACIONAL Y VIDA RURAL}

Al comparar patologías relacionadas con la realización de tareas repetitivas y físicamente exigentes entre poblaciones rurales y urbanas hay que tener presente que, tal como parecen demostrar diversos estudios clínicos, el esqueleto es más capaz de desarrollar adaptaciones durante la etapa de crecimiento (Huang et al. 2003). Por este motivo, es posible que aquellos individuos que se inicien en tareas de alta intensidad en etapas más jóvenes desarrollen una mayor robustez y masa ósea que, a su vez, propicien una menor tasa de aparición de estas patologías (Knüsel 1993).

Aunque la distribución de SN en la muestra estudiada se asemeja más a las observadas en otras necrópolis rurales, se detectan frecuencias similares en medios urbanos islámicos de la península Ibérica (fig. 14). De un modo parecido, OA es también una patología común en otras colecciones islámicas peninsulares $\mathrm{y}$, coincidiendo con la muestra observada, se localiza principalmente en la columna vertebral (Barrachina 2004-2005; Gutiérrez et al. 2016; Sánchez-Aparcero et al. 2020). Las frecuencias observadas en las extremidades de la colección estudiada son similares a las halladas en la población rural de Maro (Sánchez-Aparcero et al. 2020). En la población también rural de la Torrecilla, se observan frecuencias similares en OA vertebral (Jiménez-Brobeil et al. 2012). Puesto que el principal factor que influye en la presencia y severidad de la artrosis es la edad, seguida de carga mecánica y movimiento y también factores genéticos, es posible que el bajo número de adultos de edad avanzada encontrados en la muestra de VDU explique en los resultados obtenidos (Creamer 1997; Weiss y Jurmain 2007; Gellhorn et al. 2013).

Las fracturas parecen ser comunes en diversas poblaciones andalusíes tanto rurales como urbanas (Lacalle y Guijo 2006; Polo et al. 2008; Aran 2012; Polo et al. 2013). Es necesario recalcar que la frecuencia observada en la población urbana del Fontanar prácticamente dobla tanto la observada en la muestra estudiada como la de Marroquíes Bajos, por lo que, con los datos actuales, no parece detectase que la vida rural implicara una mayor exposición a accidentes según las técnicas empleadas (fig. 14).

Por lo tanto, los resultados obtenidos en la muestra estudiada para SN y OA parecen compatibles con los hallados en otras necrópolis rurales. En el caso de las poblaciones urbanas, no se observan grandes diferencias en la distribución de $\mathrm{SN}$, aunque no ha sido posible establecer esta comparativa con OA. Las fracturas observadas en la muestra estudiada parecen ser en su mayoría atribuibles a accidentes. 


\section{CONDICIONES DE VIDA RESPECTO A OTRAS COLECCIONES ANDALUSÍES}

Tal como se ha dicho anteriormente, no se han encontrado patrones que individualicen claramente la colección estudiada sobre las colecciones islámicas de comparación disponibles. Sin embargo, las frecuencias de DC observadas en VDU (fig. 6) son más altas que las observadas en las colecciones de Xàtiva, el Fontanar y San Nicolás (fig. 14). Aunque DC es una patología oral de origen multifactorial que no se ve afectada solamente por la dieta (Burakoff 2003; Silk et al. 2008; Hillson 2019), varios autores han propuesto su relación con un mayor consumo de carbohidratos (Keenleyside 2008; Ritter et al. 2009; Cucina et al. 2011). Es por esto que las frecuencias obtenidas en VDU podrían ser indicativas de un consumo de alimentos ricos en estas moléculas: los cereales y la fruta. Los datos historiográficos referentes a la dieta andalusí de esta región, así como los económicos que se tienen acerca de VDU en época medieval, mencionan el protagonismo de los cereales en la economía (Bolens 1990; Guichard 2001: 307; García Sánchez 2011). Recientemente, estudios carpológicos centrados en ámbitos principalmente urbanos del levante peninsular, con la excepción del estudio de Carrión et al. (2019), confirman una mayor representación de los cereales en el registro, aunque también documentan la presencia de legumbres y árboles frutales (Alonso et al. 2014; Kirchner et al. 2014; Carrión et al. 2019; Peña-Chocarro et al. 2019).

Los parámetros observados (SN, OA o fracturas) constituyen indicadores de acciones repetitivas o del riesgo de sufrir accidentes (e.g. caídas) tal como indican estudios clínicos e interpretan publicaciones basadas en poblaciones arqueológicas (Jurmain 1999: 3-5; Faccia y Williams 2008; Üstündağ 2009; Waldron 2009: 152; Dar et al. 2010; Novak y Šlaus 2011; Burke 2012). Como se ha dicho anteriormente, las frecuencias observadas en VDU no se distinguen sobremanera respecto a otras colecciones andalusíes donde estos parámetros también se han estudiado.

Respecto a las condiciones de vida en la infancia, éstas se pueden abordar desde la estatura, ya que la talla alcanzada depende de las condiciones durante el crecimiento, LEH y CO, que marcan periodos de estrés metabólico durante el desarrollo. No obstante, somos conscientes que los factores genéticos influyen en la estatura adulta por lo que no se trata de una relación simple de causa-efecto con la nutrición. El hecho de haber observado un dimorfismo sexual 1) similar al de otras poblaciones andalusíes y 2) considerado dentro de los rangos esperados en poblaciones modernas, se puede vincular a la existencia de un entorno poco adverso ya que la falta de dimorfismo sexual se vincula a la existencia de un entorno adverso durante la etapa de desarrollo del individuo (Bogin et al. 2017). Las frecuencias de individuos con marcas de LEH observadas en la población adulta son ligeramente más elevadas si se comparan con otras poblaciones islámicas (fig. 14), lo que indica que los individuos que sobrevivieron hasta la edad adulta pasaron por uno o varios periodos de estrés en la infancia. Más de la mitad de los individuos no adultos presentan marcas de $\mathrm{CO}$, mientras que los esqueletos adultos muestran frecuencias bajas si se comparan con otras poblaciones andalusíes (fig. 14).

Por lo tanto, las frecuencias de $\mathrm{CO}$ en la muestra adulta sugieren que se trata de una población con menos periodos de estrés durante la infancia. La presencia de dimorfismo sexual en la estatura apoya esta interpretación. Sin embargo, las frecuencias de LEH se presentan como mayores con respecto otras colecciones peninsulares islámicas. Diversos estudios han identificado que $\mathrm{CO}$ y LEH no presentan coocurrencia, especialmente en individuos adultos, y se ha propuesto que los patógenos que las provocarían, juntamente con una posible malnutrición y un sistema inmunológico debilitado, harían que el individuo fuera menos resistente ante posibles enfermedades adicionales y un mayor estrés (Obertová y Thurzo 2007; Liebe-Harkort 2012). Es difícil establecer una causa para las frecuencias de CO observadas en la muestra no-adulta, puesto que no hay suficientes poblaciones de comparación. Con todo, se podría interpretar que esta población rural tenía un acceso a los recursos alimentarios sujetos a limitaciones, pero en todo caso, posiblemente estas serían menores que en otras poblaciones islámicas. También que estaba menos sujeta a estrés derivado de enfermedades como la anemia, parásitos u otros causantes de estrés nutricional durante la infancia puesto que, aunque sus etiologías aún están discutidas, LEH y $\mathrm{CO}$ siguen considerándose como un buen indicador para estimar la salud y estatus nutricional durante el crecimiento en las poblaciones arqueológicas (Scott y Hoppa 2018; Hillson 2019; Pilloud y Schwitalla 2020). 


\section{CONCLUSIONES}

La mayoría de los parámetros estudiados no muestran diferencias por sexo. Sin embargo, se han detectado diferencias entre la población masculina y la femenina con respecto a la presencia de nódulos de Schmörl en los individuos. Normalmente se vinculan a una división de la actividad basada en el sexo, en la que los individuos masculinos llevarían a cabo tareas más demandantes (Faccia y Williams 2008; Üstündağ 2009; Dar et al. 2010; Novak y Šlaus 2011; Burke 2012). Aunque esta observación concuerda con las referencias historiográficas sobre la división del trabajo en la vida diaria de al-Ándalus y la vinculación de la mujer al ámbito doméstico, deben tenerse en cuenta cuatro puntos: 1) no existe un estudio que englobe los resultados de esta patología, u otras, en diferentes colecciones andalusíes y una muestra suficientemente representativa por edades; 2) no hay información acerca de la distribución de las tareas ocupacionales en el mundo rural según el sexo; 3) en la muestra adulta de la colección estudiada se ha detectado que la mayoría de individuos mayores de 30 años son de sexo masculino, lo que podría estar influyendo en los resultados observados; 4) otras patologías como la OA no muestran unas claras diferencias por sexo.

VDU presenta frecuencias similares en las patologías traumáticas y degenerativas estudiadas cuando éstas se comparan con las obtenidas en otras muestras de contextos rurales islámicos. En el estudio de las diferencias entre el ámbito rural y urbano, no se observan grandes variaciones entre las patologías de origen traumático de esta colección ni de otras islámicas. Además de ello, las fracturas observadas se pueden vincular a accidentes por lo que no son atribuibles a violencia interpersonal. Sobre las patologías degenerativas, no se han podido establecer comparaciones con base estadística con colecciones urbanas. En la misma línea, aunque la frecuencia de LEH es elevada, los marcadores vinculados a fases de estrés durante el crecimiento (CO) y la estatura no distinguen claramente la población de VDU del resto de las poblaciones andalusíes con las que se compara. Por eso, aunque es posible que sufriera periodos de estrés durante la infancia, su ocurrencia no fue extraordinaria. Sin embargo, VDU se distingue por presentar frecuencias de caries dental elevadas, que podrían estar vinculadas a un mayor consumo de carbohidratos cosa que las fuentes escritas ya atestiguan. Esta patología dental es en todo caso multifactorial y se deberá profundizar estimando la dieta de los individuos mediante métodos complementarios tales como análisis de isótopos estables.

\section{NOTAS}

1. El término hiṣn designa, en las fuentes andalusíes, principalmente a las fortificaciones rurales aunque puede corresponderse con diversas soluciones constructivas y de habitación (Bazzana et al. 1988). En general, ha sido adoptado por la historiografía para designar esta variedad de fortificaciones.

2. Algunos autores fechan aquellas fosas con orientación E-O, en vez de la usual NE-SO, en el periodo califal puesto que se alinearían con la qibla de la mezquita de Córdoba (Duarte et al. 2019).

3. Por estilo de vida se entienden las actividades que una persona o grupo suele hacer, incluida su dieta, orientaciones conductuales y perfil de acción (v.g. tareas ocupacionales) (Sniehotta $e t$ al. 2005; Haenfler et al. 2012).

4. El concepto estrés ha sido ampliamente debatido (Hubbe $\mathrm{et} \mathrm{al}$. 2018; Klaus 2014; Larsen 2015). En bioarqueología, evalúa las limitaciones ambientales, el potencial de amortiguadores culturales y la capacidad del individuo para resistir, y se manifiesta como disrupciones en el crecimiento, la presencia de ciertas patologías, o la propia muerte del individuo (Goodman et al. 1984). Se utiliza, además, para hacer inferencias acerca del estado de salud, comportamiento y adaptaciones de la población estudiada (Larsen 2015). En el caso del estrés ocupacional, se trata de inferencias relacionadas con la cantidad, y en ocasiones el tipo, de carga relacionada con la actividad física de una población.

5. Comunicación personal de M. L. Rovira a Júlia Olivé-Busom entre febrero y abril de 2018.

6. Para el hombro se ha tenido en cuenta la epífisis proximal del húmero, la cavidad glenoide y la epífisis clavicular; para el codo el húmero distal y el radio y cúbito proximales; para la muñeca el cúbito y radio distales; para la cadera el fémur proximal y el acetábulo; para la rodilla el fémur distal, la tibia proximal y la rótula; para el tobillo la tibia y el peroné distales.

\section{AGRADECIMIENTOS}

Los autores desean expresar su agradecimiento al Museu Arqueològic Municipal de la Vall d'Uixó, que ha cedido los materiales de estudio.

Júlia Olivé-Busom está financiada por un contrato FPU (FPU17 / 02934) otorgado por el Ministerio de Ciencia, Innovación y Universidades. Olalla López-costas está financiada por el Ministerio de Ciencia, Innovación y Universidades (JIN Project PID2019111683RJ-100). El proyecto Órdenes agrarias y conquistas ibéricas (siglos XII-XVI). Estudios desde la arqueología histórica (HAR201782157-P) concedido por el Ministerio de Ciencia, Innovación y Universidades ha financiado esta investigación. 


\section{BIBLIOGRAFÍA}

ALEXANDER, M. M.; GERRARD, C. M.; GUTIÉRREZ, A.; MILLARD, A. R. (2015): Diet, society, and economy in late medieval Spain: Stable isotope evidence from Muslims and Christians from Gandía, Valencia, American Journal of Physical Anthropology 156 (2), 263-273. DOI: https://doi.org/10.1002/ajpa.22647

ALFONSO, J. (2003): Memòria excavació arqueològica en carrer Soroller $n^{\circ} 5$ (Onda, Castelló), Inédito.

ALFONSO, J.; OLLER, A.; HERNÁNDEZ, R. (2006). Informe Preliminar excavación arqueológica de urgencia. Pl. El Pla, $n^{\circ}$ 1. Nova Casa de la Vila (Onda, Castellón), Inédito.

ALONSO, N.; ANTOLÍN, F.; KIRCHNER, H. (2014): Novelties and legacies in crops of the Islamic period in the northeast Iberian Peninsula: The archaeobotanical evidence in Madîna Balagî, Madîna Lârida, and Madîna Turțûša, QuaInt 346, 149-161.

DOI: https://doi.org/10.1016/j.quaint.2014.04.026

ARAN, A. (2012): La población de "Marroquíes Bajos": Reconstrucción de la vida biológica, social y cultural de la necrópolis musulmana de Jaén, Estrat crític: Revista d'Arqueologia 6, 68-84.

ARQUER, N.; COCH, C. (2017): Intervenció arqueològica d'urgència a la necròpolis andalusina del Palmar II (Borriol, Plana Alta), QPAC 35, 203-211.

ÁVILA, M. L. (1995): La estructura de la familia en al-Andalus, Casas y Palacios de al-Andalus (J. Navarro, ed.), Barcelona, 33-37.

BARCELÓ, M. (1997): Assaig d'identificació del rastre dels assentaments de la immigració berber més primerenca, El curs de les aigües. Treballs sobre els pagesos de Yâbisa (2906333 H/902-1235 dC) (M. Barceló, ed.), Eivissa, 9-28.

BARRACHINA, A. (2004-05): La necròpolis islàmica de la plaça de l'Almudín. Sogorb (Alt Palància): estudi antropològic i cronològic, QPAC 24, 281-294.

BAZZANA, A. (1996): Un Hisn valenciano: Shûn (Uxó) en la Vall d'Uixó (Castellón) I, QPAC 17, 445-475.

BAZZANA, A. (1999): Un Hisn valenciano: Shûn (Uxó) en la Vall d'Uixó (Castellón) II, QPAC 20, 317-346.

BAZZANA, A.; GUICHARD, P. (1981): Irrigation et société dans l'Espagne orientale au Moyen Age, MOM Éditions 2 (1), 115-140.

BAZZANA, A.; CRESSIER, P.; GUICHARD, P. (1988): Les châteaux ruraux d'al-Andalus: histoire et archéologie des hușūn du sud-est de l'Espagne, Madrid.

BOGIN, B.; SCHEFFLER, C.; HERMANUSSEN, M. (2017): Global effects of income and income inequality on adult height and sexual dimorphism in height, $A J H B 29$ (2), e22980. DOI: https://doi.org/10.1002/ajhb.22980

BOLENS, L. (1990): L'Andalousie du quotidien au sacré. XI-XII siècles, Norfolk.
BRANDI, A. (1992): Paleodemografía y paleopatología dental en la población hispanomusulmana de Murcia. s. XI-XIII, Tesis doctoral. Universidad Autónoma de Madrid.

BROOKS S. T.; SUCHEY, J. M. (1990): Skeletal age determination based on the os pubis: a comparison of Acsádi-Nemeskéri and Suchey-Brooks methods, Human Evolution 5, 227238.

DOI: https://doi.org/10.1007/ BF02437238

BUIKSTRA, J. E.; UBELAKER, D. H. (1994): Standards for data collection from human skeletal remains, Fayetteville.

BURAKOFF, R. P. (2003): Preventive dentistry: current concepts in women's oral health, Primary Care Update for OB/GYNS 10 (3), 141-146.

DOI: https://doi.org/10.1016/S1068-607X(03)00021-0

BURKE, K. L. (2012): Schmorl's nodes in an American military population: Frequency, Formation, and Etiology, Journal of Forensic Sciences 57 (3), 571-577.

DOI: https://doi.org/10.1111/j.1556-4029.2011.01992.x

CABANILLAS, M. I. (2012): La mujer en Al-Ándalus, Actas del IV Congreso Virtual sobre historia de las mujeres, Jaén, 3-19.

CAMPO, M. (2015): Paleopatología de la columna vertebral en la población hispanomusulmana de San Nicolás, (Murcia, S. XI-XIII), Tesis doctoral. Universidad Autónoma de Madrid.

CARRIÓN, Y.; PÉREZ-JORDÀ, G.; XIMÉNEZ DE EMBÚN, M. T. (2019): La explotación agrícola y forestal en el entorno del Cabezo Pardo (San Isidro-Granja de Rocamora, Alicante): los restos vegetales de los niveles emirales, MARQ: arqueología y museos 10, 67-78.

CHAMBERLAIN, A. (2009): Archaeological demography, Human Biology 81 (3), 275-286.

DOI: https://doi.org/10.1017/S0959774318000380

CREAMER, P.; HOCHBERG, M. C. (1997): Osteoarthritis, Lancet 350, 503-508. DOI: https://doi.org/10.1136/pmj.79.933.377

CUCINA, A.; CANTILlO, C. P.; SOSA, T. S.; TIESLER, V. (2011): Carious lesions and maize consumption among the Prehispanic Maya: an analysis of a coastal community in northern Yucatan, AJPA 145 (4), 560-567.

DOI: https://doi.org/10.1002/ajpa.21534

CURTIS, D. R.; ROOSEN, J. (2017): The sex-selective impact of the Black Death and recurring plagues in the Southern Netherlands, 1349-1450, AJPA 164 (2), 246-259. DOI: https://doi.org/10.1002/ajpa.23266

DAR, G.; MASHARAWI, Y.; PELEG, S.; STEINBERG, N.; MAY, H.; MEDLEJ, B.; PELED, N.; HERSHKOVITZ, I. (2010): Schmorl's nodes distribution in the human spine and its possible etiology, European Spine Journal 19 (4), 670-675.

DOI: https://doi.org/10.1007/s00586-009-1238-8

DEWITTE, S. N. (2010): Age patterns of mortality during the Black Death in London, AD 1349-1350, JAS 37 (12), 3394-3400. DOI: https://doi.org/10.1016/j.jas.2010.08.006 
DJILALI, K. (2018): Estudio antropológico de las necrópolis visigoda e islámica de La Almoina (Valencia), Jornades d'Arqueologia de la Comunitat Valenciana (C. Matamoros, F. E. Tendero, L. Alapont, J. Alfonso, eds.), València, 205-216.

DUARTE, F. X. (2009): Arqueología funeraria en Morella (Els Ports, Castelló): 1994-2007, Actas del IX Congreso Nacional de Paleopatología (M. Polo, E. García Prosper, eds.) Morella, 79-96.

DUARTE, F. X.; PÉREZ, R.; ARASA, F. (2019): Memòria científica: La Moleta dels Frares, Forcall (Ports, Castelló), Inédito.

DJURIĆ, M. P.; ROBERTS, C. A.; RAKOČEVIĆ, Z. B.; DJONIĆ, D. D.; LEŠIĆ, A. R. (2006): Fractures in late medieval skeletal populations from Serbia, AJPA 130 (2), 167-178. DOI: https://doi.org/10.1002/ajpa.20270

ESQUILACHE, F. (2018): Els constructors de l'Horta de València, València.

FACCIA, K. J.; WILLIAMS, R. C. (2008): Schmorl's nodes: clinical significance and implications for the bioarchaeological record, International Journal of Osteoarchaeology 18(1), $28-44$.

DOI: https://doi.org/10.1002/oa.924

GARCÍA GARCÍA, H. (1982): Notas para la historia de Vall d'Uixó, Vall d'Uixó.

GARCÍA SÁNCHEZ, E. (2011): Alimentación y paisajes agrícolas en al-Andalus, Ambienta: La revista del Ministerio de Medio Ambiente 95, 64-76.

GELLHORN, A. C.; KATZ, J. N.; SURI, P. (2013): Osteoarthritis of the spine: the facet joints, NRRheum 9 (4), 216-224. DOI: https://doi.org/10.1038/nrrheum.2012.199

GLICK, T. F. (2007): Paisajes de conquista: Cambio cultural y geográfico en la España medieval, València.

GOODMAN, A. H.; MARTIN, D. L.; ARMELAGOS, G. J. (1984): Indications of stress from bone and teeth, Paleopathology at the Origins of Agriculture (M. N. Cohen, G. J. Armelagos, eds.), Nueva York, 13-49.

GRENBY, T. H.; ANDREWS, A. T.; MISTRY M.; WILLIAMS, R. J. H. (2001): Dental caries-protective agents in milk and milk products: investigations in vitro, Journal of Dentistry 29 (2), 83-92.

DOI: https://doi.org/10.1016/S0300-5712(00)00061-0

GUICHARD, P. (1976): Al-Andalus. Estructura antropológica de una sociedad islámica en occidente, Barcelona.

GUICHARD, P. (2001): Al-Ándalus frente a la conquista cristiana: los musulmanes de Valencia, siglos XI-XIII, Valencia.

GUICHARD, P. (2016): The population of the region of Valencia during the first two centuries of Muslim domination, The Formation of al-Andalus, Part 1: History and Society (M. Marín, ed.), Abingdon, 129-182.

GUINOT, E. (1993): Sobre la depredació dels drets dels sarraïns valencians per part de les comunitats cristianes. Un exemple de la baronia d'Arenós a inicis del segle XV, Saitabi 43 , $1-10$.
GUTIÉRREZ, F. J.; LALIENA, C.; PINA, M. (2016): La maqbara medieval de Tauste, primeras investigaciones, Actas del I Coloquio de Arqueología y Patrimonio de Aragón, Zaragoza, 433-442.

HAENFLER, R.; JOHNSON, B.; JONES, E. (2012): Lifestyle movements: Exploring the intersection of lifestyle and social movements, Social Movement Studies 11(1), 1-20. DOI: $10.1080 / 14742837.2012 .640535$

HILLSON, S. (1996): Dental anthropology, Cambridge.

HILLSON, S. (2001): Recording dental caries in archaeological human remains, International Journal of Osteoarchaeology 11(4), 249-289.

DOI: https://doi.org/10.1002/oa.538

HILLSON, S. (2019): Dental pathology, Biological Anthropology of the Human Skeleton (M. A. Katzenberg, A.L. Grauer, eds.), Hoboken, 295-334.

DOI: https://doi.org/10.1002/9781119151647.ch9

HUANG, T. H.; LIN, S. C.; CHANG, F. L.; HSIEH, S. S.; LIU, S. H.; YANG, R. S. (2003): Effects of different exercise modes on mineralization, structure, and biomechanical properties of growing bone, Journal of Applied Physiology 95(1), 300-307.

DOI: https://doi.org/10.1152/japplphysiol.01076.2002

HUBBE, M.; GREEN, M. K.; CHEVERKO, C. M.; NEVES, W. A. (2018): Brief communication: A re-evaluation of the health index of southern Brazilian shellmound populations, AJPA 165 (2), 353-362.

DOI: https://doi.org/10.1002/ajpa.23346

IŞCAN, M.; LOTH, S. (1986a): Determination of Age from the Sternal Rib in White Males: A Test of the Phase Method, JFS 31 (1), 122-132.

DOI: https://doi.org/10.1520/JFS11866J

IŞCAN, M. Y.; LOTH, S. R. (1986b): Determination of age from the sternal rib in white females: a test of the phase method, JFS 31 (3), 990-999.

DOI: https://doi.org/10.1520/JFS11107J

JIMÉNEZ-BROBEIL, S.; ROCA-RODRÍGUEZ, M.; AL OUMAOUI, I.; DU SOUICH, P. (2012): Vertebral pathologies and related activity patterns in two mediaeval populations from Spain, Collegium antropologicum 36 (3), 1019-1025.

JUDD, M. A.; ROBERTS, C. A. (1999): Fracture trauma in a medieval British farming village, AJPA 109 (2), 229-243.

DOI: https://doi.org/10.1002/(SICI)1096-8644(199906) 109:2<229::AID-AJPA7>3.0.CO;2-Y

JURMAIN, R. (1999): Stories from the Skeleton: Interpreting the Remains of the Past, San José.

KEENLEYSIDE, A. (2008): Dental pathology and diet at Apollonia, a Greek colony on the Black Sea, International Journal of Osteoarchaeology 18(3), 262-279. DOI: https://doi.org/10.1002/oa.934

KIRCHNER, H.; NAVARRO, C. (1993): Objetivos, métodos y práctica de la arqueología hidràulica, Arqueología y Territorio Medieval 1, 159-182. 
KIRCHNER, H.; VIRGILI, A.; ANTOLÍN, F. (2014): Un espacio de cultivo urbano en al-Andalus: Madîna Turtûsa (Tortosa) antes de 1148, Historia agraria: Revista de agricultura e historia rural 62, 11-45.

KLAUS, H. D. (2014): Frontiers in the bioarchaeology of stress and disease: Cross-disciplinary perspectives from pathophysiology, human biology, and epidemiology, AJPA 155 (2), 294-308.

DOI: https://doi.org/10.1002/ajpa.22574

KNÜSEL, C. J. (1993): On the biomechanical and osteoarthritic differences between hunter-gatherers and agriculturalists, AJPA 91 (4), 523-525.

DOI: https://doi.org/10.1002/ajpa.1330910409

KNÜSEL, C. J.; GÖGGEL, S.; LUCY, D. (1997): Comparative degenerative joint disease of the vertebral column in the medieval monastic cemetery of the Gilbertine Priory of St. Andrew, Fishergate, York, England, AJPA 103 (4), 481-495. DOI: https://doi.org/10.1002/(SICI)1096-8644(199708) 103:4<481::AID-AJPA6>3.0.CO;2-Q

KUBEHL, K.; TEMPLE, D. H. (2020): Reproductive life histories influence cariogenesis: Exploring sex-specific variation in dental caries and survivorship in the human past, AJPA 172 (3), 376-385.

DOI: https://doi.org/10.1002/ajpa.24034

KYRIAKOU, X. P.; MARQUEZ-GRANT, N.; LANGSTAFF, H.; SAMUELS, C.; SPRINGS PACELLI, C.; CASTRO, J.; KRANIOTI, E. (2012): The human remains from the medieval Islamic cemetery of Can Fonoll, Ibiza, Spain: Preliminary results, Proceedings of the twelfth annual conference of the British Association for Biological Anthropology and Osteoarchaeology (P. D. Mitchell, J. Buckberry, eds.), Oxford, 87-101.

LACALLE, R.; GUIJO, J. M. (2006): Análisis antropológico de la población islámica califal de El Fontanar, AAC 17 (2), 291-316.

LALUEZA-FOX, C. (1998): Stature and sexual dimorphism in ancient Iberian populations, Homo 49, 260-272.

LARSEN, C. S. (2015): Bioarchaeology: Interpreting Behavior from the Human Skeleton, Cambridge.

LIEBE-HARKORT, C. (2012): Cribra orbitalia, sinusitis and linear enamel hypoplasia in Swedish Roman Iron Age adults and subadults, International Journal of Osteoarchaeology 22(4), 387-397.

DOI: https://doi.org/10.1002/oa.1209

LÓPEZ-MORANGO, C. (2020): Estudio comparativo de la calidad de vida en la Edad Media, en poblaciones musulmanas y cristianas de la Península Ibérica, a través de la Antropología Dental, Tesis doctoral. Universidad de Granada.

LOVEJOY, C. O.; MEINDL, R. S.; PRYZBECK, T. R.; MENSFORTH, R. P. (1985): Chronological metamorphosis of the auricular surface of the ilium. A new method for the determination of adult skeletal age at death, AJPA 68 (1), 15-28 DOI: https://doi.org/10.1002/ajpa.1330680103
LOVELL, N. C.; GRAUER, A. L. (2019): Analysis and Interpretation of Trauma in Skeletal Remains, Biological Anthropology of the Human Skeleton (M.A. Katzenberg, A.L. Grauer, eds.), Hoboken, 335-384. DOI: https://doi.org/10.1002/9781119151647.ch10

MANOUVRIER, L. (1893): La determination de la taille d'apres les grands os des membres, Mémoires de la Société d'Anthropologie de Paris 4, 347-402.

MARTIN, D. L.; HARROD, R. P. (2015): Bioarchaeological contributions to the study of violence, AJPA 156, 116-145. DOI: https://doi.org/10.1002/ajpa.22662

MCKINLEY, J. L. (2004): Compiling a skeletal inventory: disarticulated and co-mingled remains, Guidelines to the Standards for Recording Human Remains (IFA Technical Paper 7) (M. Brickley, J. L. McKinley eds.), Southampton, 14-17.

MELCHOR, J. M.; BENEDITO, J. (2018): Estudio general de las maqābir de la Madīna Buryena (Burriana, Castellón), QPAC 36, 203-216.

MENDONÇA, M. C. (2000): Estimation of height from the length of long bones in a Portuguese adult population, AJPA 112 (1), 39-48.

DOI: https://doi.org/10.1002/(SICI)1096-8644(200005) 112:1<39::AID-AJPA5>3.0.CO;2-\%23

MEYER, A.; STEYN, M. (2016): Chinese Indentured Mine Labour and the Dangers Associated with Early 20th Century Deeplevel Mining on the Witwatersrand Gold Mines, South Africa, International Journal of Osteoarchaeology 26 (4), 648-660.

DOI: https://doi.org/10.1002/oa.2455

MIRA, J. A.; CARRIÓN, Y.; MORALES-PÉREZ, J. V.; ORTEGA, J. R. (2017): Nuevas aportaciones sobre el aprovechamiento faunístico y vegetal en el Castell de Castalla (Alicante, España) durante los siglos XI-XV, Arqueología y Territorio Medieval 24, 291-33.

MOLERO, I. (2017): Estudio bioantropológico de la maqbara andalusí de Toledo (siglos VIII-XI). Apuntes sobre el proceso de islamización, Tesis doctoral. Universidad Autónoma de Madrid.

NEGRE, M. C.; FORNER, A. B.; PUCHALT, F. J.; VILLALAÍN, J. D. (2003): Estudio antropológico y paleopatológico de Sant Pere (Xátiva), Actas del VI Congreso Nacional de Paleopatología (M. Campo Martín, F. J. Robles Rodríguez eds.), Madrid, 220-225.

NOVERINT (2017): Informe preliminar. Seguimiento arqueológico. Actuación de mejora de la calle Virgen del Rosario (Morella), Inédito.

NOVAK, M.; ŠLAUS, M. (2011): Vertebral pathologies in two early modern period (16th-19th century) populations from Croatia, AJPA 145 (2), 270-281. DOI: https://doi.org/10.1002/ajpa.21491

OBERTOVÁ, Z.; THURZO, M. (2007): Relationship between cribra orbitalia and enamel hypoplasia in the early medieval 
Slavic population at Borovce, Slovakia, International Journal of Osteoarchaeology 18 (3), 280-292.

DOI: https://doi.org/10.1002/oa.937

PEARSON, K. (1899): IV. Mathematical contributions to the theory of evolution.-V. On the reconstruction of the stature of prehistoric races, Philosophical Transactions of the Royal Society 192 (193), 169-244.

DOI: https://doi.org/10.1098/rsta.1899.0004

PEÑA-CHOCARRO, L.; PÉREZ-JORDÀ, G.; ALONSO, N.; ANTOLÍN, F.; TEIRA-BRIÓN, A.; TERESO, J.P.; MOYA, E. M. M.; REYES, D. L. (2019): Roman and medieval crops in the Iberian Peninsula: a first overview of seeds and fruits from archaeological sites, QuaInt 499, 49-66. DOI: https://doi.org/10.1016/j.quaint.2017.09.037

PÉREZ ORDÓÑEZ, P. (2018): Vida cotidiana: la vivienda andalusí a través de la Arqueología en la provincia de Málaga, El islam. Presente de un pasado medieval: XXVIII Semana de Estudios Medievales (E. López Ojeda, coord.), Nájera, 205-250.

PILLOUD, M. A.; SCHWITALLA, A. W. (2020): Re-evaluating traditional markers of stress in an archaeological sample from central California, JAS 116, 105102.

DOI: https://doi.org/10.1016/j.jas.2020.105102

POLO, M.; CRUZ, E.; COCH, C. (2008): Bioantropología de la necrópolis hispano-musulmana del nuevo hogar 'Verge del Lledó'(Castellón), Butlletí de la Societat Castellonenca de Cultura 84, 475-488.

POLO, M.; GARCÍA-PRÓSPER, E.; MELCHOR, J. M.; BENEDITO, J. (2013): Paleopatología en tres conjuntos funerarios medievales de Burriana (Castellón), Actas del XI Congreso Nacional de Paleopatología (A. Malgosa, A. Isidro, P. Ibáñez-Gimeno, G. Prats-Muñoz, eds.), Barcelona, 573-598.

REDFERN, R. C.; DEWITTE, S. N.; PEARCE, J.; HAMLIN, C.; DINWIDDY, K. E. (2015): Urban-rural differences in Roman Dorset, England: A bioarchaeological perspective on Roman settlements, AJPA 157 (1), 107-120. DOI: https://doi.org/10.1002/ajpa.22693

RITTER, A. V.; GRIPPO, J. O.; COLEMAN, T. A.; MORGAN, M. E. (2009): Prevalence of carious and non-carious cervical lesions in archaeological populations from North America and Europe, Journal of Esthetic and Restorative Dentistry 21 (5), 324-334.

DOI: https://doi.org/10.1111/j.1708-8240.2009.00285.x

RODRIGUES, A. C. (2013): A maqbara de Shantarîn: Enfermidade e saúde numa amostra esquelética de adultos, Tesis de máster. Universidad de Coimbra.

ROUSHAM, E. K.; HUMPHREY, L. T. (2002): The dynamics of child survival, Human Population Dynamics. Cross-Disciplinary Perspectives (H. Macbeth, P. Collison, eds.), Cambridge, 124-140.

SÁNCHEZ-APARCERO, B.; ALEMÁN, I.; BOTELLA, M. C. (2020): The necropolis of Maro (Málaga, Spain): an anthropological study, AAS 12 (7), 1-17. DOI: https://doi.org/10.1007/s12520-020-01092-4
SANDERS, K. M.; NICHOLSON, G. C.; UGONI, A. M.; SEEMAN, E.; PASCO, J. A.; KOTOWICZ, M. A. (2002): Fracture rates lower in rural than urban communities: the Geelong Osteoporosis Study, JECH 56 (6), 466-470. DOI: http://dx.doi.org/10.1136/jech.56.6.466

SCHAEFER, M.; BLACK, S. M.; SCHEUER, L. (2009): Juvenile osteology, San Diego.

SCHWEICH, M.; KNÜSEL, C. (2003): Bio-cultural effects in medieval populations, EHB 1(3), 367-377. DOI: https://doi.org/10.1016/j.ehb.2003.09.002

SCOTT, A. B.; HOPPA, R. D. (2018): The subtleties of stress: A comparative analysis of skeletal lesions between the Medieval and post-Medieval Black Friars cemetery population (13th to 17th centuries), International Journal of Osteoarchaeology 28 (6), 695-702.

DOI: https://doi.org/10.1002/oa.2691

SELMA, S. (1991): El molí hidràulic de farina I: L'organització de l'espai rural andalusí. Dos exemples d'estudi arqueològic espacial a la serra d'Espadà (Castelló), Mélanges de la Casa de Velázquez 27 (1), 65-100.

SHATZMILLER, M. (1993): Labour in the medieval Islamic world, Leiden.

SILK, H.; DOUGLASS, A. B.; DOUGLASS, J. M.; SILK, L. (2008): Oral health during pregnancy, American Family Physician 77 (8), 1139-1144.

ŠLAUS, M.; NOVAK, M.; BEDIĆ, Ž.; STRINOVIĆ, D. (2012): Bone fractures as indicators of intentional violence in the eastern adriatic from the antique to the late medieval period (2nd-16th century AD), AJPA 149 (1), 26-38. DOI: https://doi.org/10.1002/ajpa.22083

SNIEHOTTA, F. F.; SCHWARZER, R.; SCHOLZ, U.; SCHÜZ, B. (2005): Action planning and coping planning for long-term lifestyle change: theory and assessment, EJSP 35 (4), 565-576. DOI: https://doi.org/10.1002/ejsp.258

SOUICH, P. (1974): La necrópolis y poblado de la Torrecilla. Estudio antropológico, Anuario de Estudios Medievales 9, 41-74.

STUART-MACADAM, P. (1991): Anaemia in Roman Britain: Poundbury Camp, Health in past societies: biocultural interpretations of human skeletal remains in archaeological contexts (H. Bush, M. Zvelebil, eds.), Oxford, 101-113.

STUART-MACADAM, P. (1998): Iron deficiency anemia: exploring the difference, Sex and gender in paleopathological perspective (A. L. Grauer, P. Stuart-Macadam, eds.), Cambridge, 45-63.

TORRÓ, J. (2007): Guerra, repartiment i colonització al regne de València (1248-1249), Repartiments a la Corona d'Aragó (segles XII-XIII) (E. Guinot, J. Torró, eds.), València, 201-276.

TORRÓ, J. (2010): Els camperols musulmans del regne de València. De la conquesta a la conversió, La Rella 23, 201-212.

TORRÓ, J. (2012): Les Regions orientals d'al-Àndalus abans de la conquesta catalanoaragonesa. Una visió general, Catalan Historical Review 5, 143-157. 
ÜSTÜNDAĞ, H. (2009): Schmörl's nodes in a post-medieval skeletal sample from Klostermarienberg, Austria, International Journal of Osteoarchaeology 19(6), 695-710.

DOI: https://doi.org/10.1002/oa.993

VERCELLOTTI, G.; PIPERATA, B. A.; AGNEW, A. M; WARREN, W. M; DUFOUR, D. L.; REINA, J. C.; BOANO, J.; JUSTUS, H. M.; LARSEN, C. S.; STOUT, S. D.; SCIULLI, P. W. (2014): Exploring the multidimensionality of stature variation in the past through comparisons of archaeological and living populations, AJPA 155 (2), 229-242.

DOI: https://doi.org/10.1002/ajpa.22552
WALDRON, T. (2009): Palaeopathology, Cambridge.

WALKER, P. L.; JOHNSON, J. R.; LAMBERT, P. M. (1988): Age and sex biases in the preservation of human skeletal remains, AJPA 76 (2), 183-188.

DOI: https://doi.org/10.1002/ajpa.1330760206

WEISS, E.; JURMAIN, R. (2007): Osteoarthritis revisited: a contemporary review of aetiology, International Journal of Osteoarchaeology 17(5), 437-450.

DOI: https://doi.org/10.1002/oa.889

WHITE, T. D.; FOLKENS, P. A. (2005): The human bone manual, London. 